\title{
Six-dimensional weak-strong simulation of head-on beam-beam compensation in the Relativistic Heavy Ion Collider
}

\author{
Y. Luo, ${ }^{*}$ W. Fischer, N. P. Abreu, X. Gu, A. Pikin, and G. Robert-Demolaize \\ Brookhaven National Laboratory, Upton, New York 11973, USA
}

(Received 5 August 2010; revised manuscript received 5 August 2011; published 18 May 2012)

\begin{abstract}
To compensate the beam-beam tune spread and beam-beam resonance driving terms in the polarized proton operation in the Relativistic Heavy Ion Collider (RHIC), we will introduce a low energy DC electron beam into each ring to collide head-on with the opposing proton beam. The device to provide the electron beam is called an electron lens. In this article, using a 6D weak-strong beam-beam interaction simulation model, we will investigate the effects of head-on beam-beam compensation with electron lenses on the proton beam dynamics for the RHIC $250 \mathrm{GeV}$ polarized proton operation. Frequency maps, dynamic apertures, and proton beam loss rates are calculated for this study. Key beam parameters involved in this scheme are varied to search for the optimum compensation condition. The sensitivities of head-on beam-beam compensation to beam imperfections and beam offsets are also studied.
\end{abstract}

DOI: $10.1103 /$ PhysRevSTAB.15.051004

PACS numbers: 29.27.Bd, 29.20.-c

\section{INTRODUCTION}

The working point in the polarized proton operation in the Relativistic Heavy Ion Collider (RHIC) is chosen to provide a good beam-beam lifetime and to maintain the proton polarization during the energy acceleration and at the physics store. The current working point is constrained between $2 / 3$ and $7 / 10$ [1,2]. $2 / 3$ is a strong third order betatron resonance. $7 / 10$ is a 10 th order betatron resonance and a depolarization resonance [3]. Experiments and simulations have shown that the beam-beam lifetime and the proton polarization are reduced when the vertical tune of the proton beam is close to $7 / 10$.

To further increase the luminosity in the polarized proton operation in RHIC, it is planned to increase the proton bunch intensity from currently $1.65 \times 10^{11}$ up to $3.0 \times 10^{11}$ with an upgraded polarized proton source [4]. However, with such a high proton bunch intensity, the linear incoherent beam-beam tune shift and the amplitude dependent beam-beam tune spread will be about 0.03 . To hold the large beam-beam tune spread in the current tune space and to compensate the large beam-beam resonance driving terms, we are planning to install head-on beambeam compensation in RHIC [5-7].

Head-on beam-beam compensation in proton colliders with electron beams was first proposed and studied by Tsyganov in 1993 [8,9]. The idea is to introduce a low energy electron beam to collide head-on with the opposing proton beam to compensate the beam-beam nonlinear effects. The device to provide the electron beam is called an

\footnotetext{
*yluo@bnl.gov
}

Published by the American Physical Society under the terms of the Creative Commons Attribution 3.0 License. Further distribution of this work must maintain attribution to the author(s) and the published article's title, journal citation, and DOI. electron lens (e-lens). A pulsed e-lens had been successfully installed and operated in the Tevatron to compensate the long-range beam-beam tune shifts [10-13].

In our current design for the RHIC head-on beam-beam compensation [14], two e-lenses are needed. One for the blue ring and one for the yellow ring. They will be installed on either side of the interaction point (IP) IP10. The two proton beams collide at IP6 and IP8 with $\beta^{*}=0.5 \mathrm{~m}$. The $\beta$ functions at the e-lenses are $10 \mathrm{~m}$. The transverse rms beam size of the proton beam at the e-lenses is $310 \mu \mathrm{m}$.

In the following, we will first present the physics of head-on beam-beam compensation. Then we introduce the beam and lattice parameters for its application in RHIC. To investigate its effects on the proton beam dynamics in RHIC, we calculate frequency maps, dynamic apertures, and proton beam loss rates. Key beam parameters involved in this scheme are varied to search for the optimum compensation condition. The sensitivity of headon beam-beam compensation to beam imperfections and beam offsets are also studied.

\section{HEAD-ON BEAM-BEAM COMPENSATION}

In this section we will present the physics of head-on beam-beam compensation with e-lenses. For illustration, we assume that both the proton bunch and the electron beam have a round Gaussian transverse distribution, which is the case in RHIC. We model the interaction between a test proton and the opposing proton bunch or electron beam as a $4 \mathrm{D}$ weak-strong beam-beam thin-lens kick.

In the following, we will calculate the linear incoherent beam-beam tune shift, the amplitude dependent beambeam tune shift, and the beam-beam resonance driving terms. We will discuss the conditions for head-on beam-beam compensation for the case of one head-on beam-beam interaction point and one e-lens in the ring. 


\section{A. Linear incoherent beam-beam tune shift}

For head-on beam-beam compensation in RHIC, we will introduce a low energy DC electron beam into each ring to collide head-on with the opposing proton bunch. The kinetic energy of electrons is $5-10 \mathrm{keV}$. The speed of the electrons is $\beta_{e} c, c$ is the speed of light. For an ultrarelativistic test proton going through one slice of the electron beam, the Lorentz forces $F_{x, y}$ are

$$
\left(\begin{array}{c}
F_{x} \\
F_{y}
\end{array}\right)_{p-e}=-\frac{n_{e} e^{2}\left(1+\beta_{e}\right)}{2 \pi \epsilon_{0} r^{2}}\left(1-e^{-r^{2} / 2 \sigma_{e}^{2}}\right)\left(\begin{array}{l}
x \\
y
\end{array}\right) .
$$

Here $r=\sqrt{x^{2}+y^{2}}, n_{e}$ is the line particle density of the electron beam, and $\sigma_{e}$ is the transverse rms beam size of the electron beam in the e-lens. $\beta_{e}$ in Eq. (1) comes from the fact that $\mathbf{B}=\vec{\beta}_{e} \times \mathbf{E} / c$, where $\mathbf{E}$ and $\mathbf{B}$ are the electric and magnetic fields generated by the electron slice.

The total transverse kicks on the test proton for one passage through the e-lens are

$$
\left(\begin{array}{c}
\Delta x^{\prime} \\
\Delta y^{\prime}
\end{array}\right)_{p-e}=-\frac{2 N_{e}^{*} r_{0}}{\gamma r^{2}}\left(1-e^{-r^{2} / 2 \sigma_{e}^{2}}\right)\left(\begin{array}{l}
x \\
y
\end{array}\right) .
$$

Here $r_{0}$ is the classic proton radius, $\gamma$ is the Lorentz relativistic factor of the test proton, and the effective electron intensity of the e-lens is $N_{e}^{*}=N_{e}\left(1+\beta_{e}\right) . N_{e}$ is the total number of electrons in the e-lens. The electron beam current is $I_{e}=e N_{e} \beta_{e} c / L_{e}, L_{e}$ is the effective length of the e-lens.

Similarly, for the proton-proton beam-beam interaction, the total transverse beam-beam kicks on an ultrarelativistic proton after one passage through a round Gaussian proton bunch are [15]

$$
\left(\begin{array}{c}
\Delta x^{\prime} \\
\Delta y^{\prime}
\end{array}\right)_{p-p}=\frac{2 N_{p} r_{0}}{\gamma r^{2}}\left(1-e^{-r^{2} / 2 \sigma_{p, \mathrm{IP}}^{2}}\right)\left(\begin{array}{l}
x \\
y
\end{array}\right) .
$$

Here $N_{p}$ is the proton bunch intensity, $\sigma_{p, \text { IP }}$ is the proton bunch's transverse rms beam size at the interaction point. Comparing Eqs. (3) and (2), the transverse kicks given by a round Gaussian proton bunch and a round Gaussian electron beam of an e-lens have the same dependence of the strong beam's particle intensity, beam size, and the test proton's coordinates, except for the sign.

To calculate the linear incoherent beam-beam tune shift for a proton with a very small amplitude, we take the approximations that $r / \sigma_{p, \mathrm{IP}} \ll 1$ and $r / \sigma_{e} \ll 1$ in Eqs. (2) and (3),

$$
\begin{aligned}
& \left(\begin{array}{c}
\Delta x^{\prime} \\
\Delta y^{\prime}
\end{array}\right)_{p-p}=\frac{N_{p} r_{0}}{\gamma \sigma_{p, \mathrm{IP}}^{2}}\left(\begin{array}{l}
x \\
y
\end{array}\right) . \\
& \left(\begin{array}{c}
\Delta x^{\prime} \\
\Delta y^{\prime}
\end{array}\right)_{p-e}=-\frac{N_{e}^{*} r_{0}}{\gamma \sigma_{e}^{2}}\left(\begin{array}{l}
x \\
y
\end{array}\right) .
\end{aligned}
$$

Therefore, the linear tune shifts due to the head-on beambeam interaction and the e-lens are

$$
\begin{gathered}
\left.\Delta Q_{x, y}\right|_{p-p}=-\frac{N_{p} r_{0} \beta_{\mathrm{IP}}^{*}}{4 \pi \gamma \sigma_{p, \mathrm{IP}}^{2}}, \\
\left.\Delta Q_{x, y}\right|_{p-e}=\frac{N_{e}^{*} r_{0} \beta_{\text {e-lens }}}{4 \pi \gamma \sigma_{e}^{2}} .
\end{gathered}
$$

Here $\beta_{\mathrm{IP}}^{*}$ and $\beta_{\mathrm{e}-\text { lens }}$ are the proton beam's betatron amplitude functions at the beam-beam interaction point and at the e-lens.

According to Eqs. (6) and (7), to cancel the linear incoherent beam-beam tune shift from one proton-proton interaction with one e-lens, we need $N_{e}^{*}=N_{p}$ and $\sigma_{e}^{2} / \beta_{\mathrm{e}-\mathrm{lens}}=\sigma_{p, \mathrm{IP}}^{2} / \beta_{\mathrm{IP}}^{*} . \quad$ Considering $\quad \sigma_{p, \mathrm{IP}}^{2} / \beta_{\mathrm{IP}}^{*}=$ $\sigma_{p \text {,e-lens }}^{2} / \beta_{\text {e-lens }}$, where $\sigma_{p, \text { e-lens }}$ is the proton bunch's rms transverse beam size at the e-lens, we have $\sigma_{e}=\sigma_{p \text {,e-lens }}$, which means the electron beam should have the same transverse beam size as the proton beam at the e-lens.

\section{B. Nonlinear beam-beam tune shift and resonance driving terms}

The beam-beam interaction and e-lenses also introduce nonlinear amplitude dependent tune shift and beambeam resonance driving terms. To calculate them, we use the Hamiltonian perturbation approach [15-20]. The Hamiltonian of the ring in action-angle variables is given by

$$
H\left(J_{x}, J_{y}, \Phi_{x}, \Phi_{y}\right)=2 \pi Q_{x} J_{x}+2 \pi Q_{y} J_{y}+\sum_{i=1}^{N} V_{i}\left(x_{n}, y_{n}\right),
$$

where $J_{x, y}$ and $\Phi_{x, y}$ are the horizontal and vertical actions and angles. $V_{i}(x, y)$ is the $i$ th beam-beam interaction potential. In our case, it can be from the proton-proton interaction or from the proton-e-lens interaction. The input variables of $V_{i}\left(x_{n}, y_{n}\right)$ in Eq. (8) are the normalized coordinates $x_{n}=$ $\sqrt{2 J_{x} \beta_{x i}} \cos \left(\Phi_{x}+\phi_{x i}\right)$ and $y_{n}=\sqrt{2 J_{y} \beta_{y i}} \cos \left(\Phi_{y}+\phi_{y i}\right)$, where $\beta_{x i, y i}$ and $\phi_{x i, y i}$ are the betatron functions and phase advances at the $i$ th beam-beam interaction point. In Eq. (8), we excluded the effects of nonlinear magnetic fields in the ring.

For simplicity, we only discuss a 2D situation and consider only horizontal motion. Then, the Hamiltonian is simplified to

$$
H\left(J_{x}, \Phi_{x}\right)=2 \pi Q_{x} J_{x}+\sum_{i}^{N} V_{i}\left(x_{n}\right) .
$$

The 2D beam-beam potential is given by

$$
V_{i}\left(x_{n}\right)=-\int_{0}^{x} \Delta x^{\prime} d x .
$$


$\Delta x^{\prime}$ is the horizontal beam-beam kick. According to Eqs. (2) and (3), for the proton-proton beam-beam interaction and proton-e-lens interaction,

$$
\begin{aligned}
& \left.\Delta x^{\prime}\right|_{p-p}=\frac{2 N_{p} r_{0}}{\gamma} \frac{1}{x}\left(1-e^{-x^{2} / 2 \sigma_{p, \mathrm{IP}}^{2}}\right), \\
& \left.\Delta x^{\prime}\right|_{p-e}=-\frac{2 N_{e}^{*} r_{0}}{\gamma} \frac{1}{x}\left(1-e^{-x^{2} / 2 \sigma_{e}^{2}}\right) .
\end{aligned}
$$

Plugging them into Eq. (10) and changing the integral variable, their potentials are [17]

$$
\begin{aligned}
\left.V\left(J_{x}, \Phi_{x}\right)\right|_{p-p}= & -\frac{N_{p} r_{0}}{\gamma} \int_{0}^{J_{x} \beta_{\mathrm{IP}}^{*} / 2 \sigma_{p, \mathrm{IP}}^{2}} \\
& \times \frac{d \alpha}{\alpha}\left(1-e^{-2 \alpha \cos ^{2}\left(\Phi_{x}+\phi_{x, p-p}\right)}\right), \\
\left.V\left(J_{x}, \Phi_{x}\right)\right|_{p-e}= & \frac{N_{e}^{*} r_{0}}{\gamma} \int_{0}^{J_{x} \beta_{\mathrm{e}-\text { lens }} / 2 \sigma_{e}^{2}} \\
& \times \frac{d \alpha}{\alpha}\left(1-e^{-2 \alpha \cos ^{2}\left(\Phi_{x}+\phi_{x, p-e}\right)}\right) .
\end{aligned}
$$

Equations (13) and (14) have the same structures and the same dependence on the beam parameters, except for the sign. With one beam-beam interaction and one e-lens in the ring, to completely cancel their potentials, we obtain the following conditions: (1) $N_{e}^{*}=N_{p}$, (2) $\sigma_{e}^{2} / \beta_{\mathrm{e}-\mathrm{lens}}=\sigma_{p, \mathrm{IP}}^{2} / \beta_{\mathrm{IP}}^{*}$, which means that the electron beam has the same transverse size as the proton beam at the e-lens, and (3) $\phi_{x, p-p}-\phi_{x, p-e}=k \pi$, where $k$ is an integer, that is, the betatron phase advances between the beam-beam and the e-lens should be multiples of $\pi$.

To first order of the strengths of beam-beam interaction and e-lens, phase advances $k \pi$ with $k$ even between the beam-beam interaction point and the e-lens may introduce dispersion beat between them. Therefore, to compensate both the beam-beam tune shift and beam-beam resonance driving terms without dispersion beat between the beambeam interaction point and the e-lens, we would like to choose odd multiples of $\pi$ phase advances between them.

The beam-beam potentials in Eq. (9) are functions of $J_{x}$ and $\Phi_{x}$. To isolate the different resonances, for a certain action $J_{x}$, we expand the beam-beam potentials into Fourier series of $\Phi_{x}$,

$$
\sum_{i}^{N} V_{i}\left(x_{n}\right)=h_{0}\left(J_{x}\right)+\sum_{n \neq 0} h_{n}\left(J_{x}\right) e^{-i n \Phi_{x}} .
$$

Then the 2D Hamiltonian is simplified to

$$
H\left(J_{x}, \Phi_{x}\right)=2 \pi Q_{x} J_{x}+h_{0}\left(J_{x}\right)+\sum_{n \neq 0} h_{n}\left(J_{x}\right) e^{-i n \Phi_{x}} .
$$

Finally, we obtain the amplitude dependent beam-beam tune shift as

$$
\Delta Q_{x}\left(J_{x}\right)=\frac{1}{2 \pi} \frac{\partial h_{0}\left(J_{x}\right)}{\partial J_{x}} .
$$

When $J_{x}=0, \Delta Q_{x}(0)$ gives the total linear incoherent beam-beam tune shift from the beam-beam interaction and e-lens. $h_{n}\left(J_{x}\right)$, where $n \neq 0$, is the beam-beam resonance driving term. $h_{n}\left(J_{x}\right)$ will drive the resonance $n Q_{x}=$ $p, p$ is an integer. With a round Gaussian beam, only even order beam-beam resonance driving terms are generated $[15,17]$. Following Refs. $[15,16]$, we also define the width of the beam-beam resonance as $d h_{n}\left(J_{x}\right) / d J_{x}$. According to Refs. [16,18,21], resonance overlapping will cause chaotic motion and reduce dynamic aperture.

In the above discussion, we assumed that the proton bunch length is much smaller than the betatron amplitude function $\beta^{*}$ at the proton-proton interaction point. However, in the RHIC $250 \mathrm{GeV}$ polarized proton operation, the proton bunch length is about $0.45 \mathrm{~m}$ which is comparable to $\beta^{*}=0.5 \mathrm{~m}$ at IP6 and IP8 [22]. We also ignored the magnetic nonlinearity [23] in the proton ring and the proton particle's longitudinal motion. To fully study the effects of head-on beam-beam compensation on the proton beam dynamics through simulation, a 6D symplectic beam-beam interaction model and 6D symplectic magnet transfer maps are needed.

\section{APPLICATION IN RHIC}

\section{A. Layout}

There are two rings in RHIC: the blue ring and the yellow ring. The proton beam in the blue ring circulates clockwise, while the proton beam in the yellow ring circulates counterclockwise. The two beams collide at IP6 and IP8. Normally the $\beta^{*}$ s at the two IPs are same. To compensate head-on beam-beam interaction in both rings, we plan to install two e-lenses, one for each ring. The e-lens in the blue ring is on the south side of IP10 and the e-lens in the yellow ring is on the north side of IP10. They are $1.5 \mathrm{~m}$ away from IP10. The effective compensation length of elenses is $2 \mathrm{~m}$. Figure 1 shows the layout of head-on beambeam compensation in RHIC.

In the operation of head-on beam-beam compensation with the e-lenses, the two proton beams will be separated vertically by $10 \mathrm{~mm}$ in the e-lenses. $10 \mathrm{~mm}$ is about $32 \sigma$ with $\beta_{\text {e-lens }}=10 \mathrm{~m}$. Operational experience and beam experiment studies show that the long-range beam-beam forces between the proton bunches and the electron beams in the e-lenses are negligible [24,25]. To stabilize the electron beam, each compensation region is surrounded by a superconducting solenoid. The magnetic field of the solenoids ranges from 3 to $6 \mathrm{~T}$. To cancel their effect on the global betatron coupling and spin dynamics for the polarized proton beams, the two solenoids are powered with opposite polarities. 


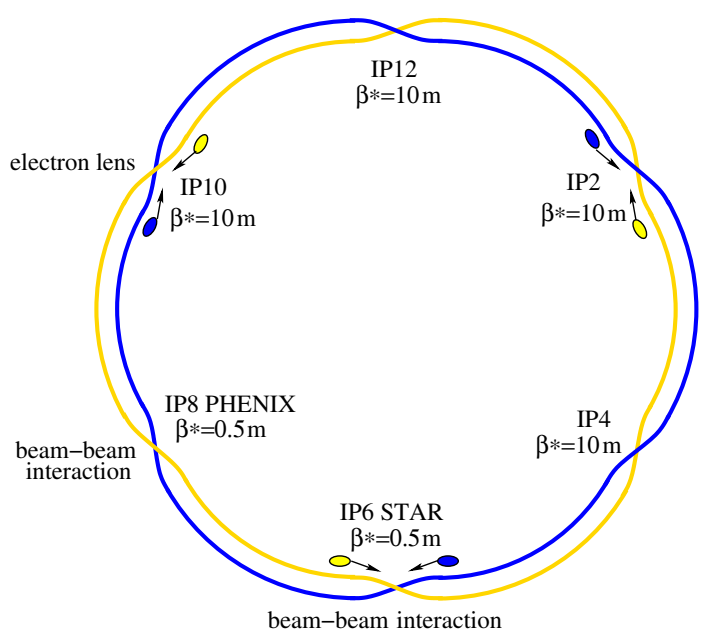

FIG. 1. Layout of head-on beam-beam compensation in RHIC. The two proton beams collide at IP6 and IP8. The e-lens in the blue ring is on the south side of IP10, and the e-lens in the yellow ring is on the north side of IP10.

\section{B. Lattice and beam parameters}

In the following simulation, we use a proposed blue ring lattice for $250 \mathrm{GeV}$ RHIC polarized proton operation. Table I lists the lattice and beam parameters. RHIC has not yet operated with $\beta^{*}=0.5 \mathrm{~m}$. $\beta^{*} \mathrm{~s}$ in the $2011 \mathrm{RHIC}$ $250 \mathrm{GeV}$ polarized proton run were $0.6 \mathrm{~m}$. A low $\beta^{*}$ lattice will increase off-momentum tune shift and off-momentum $\beta$ beat and reduce the momentum aperture. In RHIC, we

TABLE I. Lattice and beam parameters used in this study.

\begin{tabular}{lc}
\hline \hline Parameter & Value \\
\hline Ring circumference & $3833.8451 \mathrm{~m}$ \\
Energy & $250 \mathrm{GeV}$ \\
Relativistic $\gamma$ & 266 \\
Working point $Q_{x, y}$ & $(28.67,29.68)$ \\
Linear chromaticities $\xi_{x, y}^{(1)}$ & $(1,1)$ \\
Second order chromaticities $\xi_{x, y}^{(2)}$ & $(2800,2900)$ \\
Normalized transverse rms emittance $\epsilon_{n}$ & $2.5 \mathrm{~mm} \mathrm{mrad}$ \\
$\beta_{x, y}^{*}$ at IP6 and IP8 & $0.5 \mathrm{~m}$ \\
$\beta_{x, y, \text { e-lens }}$ at the e-lens & $10.0 \mathrm{~m}$ \\
Transverse rms beam size at IP6 and IP8 & $68 \mu \mathrm{m}$ \\
Transverse rms beam size at e-lens & $310 \mu \mathrm{m}$ \\
Phase advances between IP6 and IP8 & $(10.6 \pi, 9.7 \pi)$ \\
Phase advances between IP8 and e-lens & $(8.5 \pi, 11.1 \pi)$ \\
Optimized phase advances & $(9.0 \pi, 11.0 \pi)$ \\
between IP8 and e-lens & \\
Harmonic number & 360 \\
rf cavity voltage & $300 \mathrm{kV}$ \\
Longitudinal rms bunch area & $0.17 \mathrm{eV} \mathrm{s}$ \\
Bucket height $\left(d p / p_{0}\right)_{\text {max }}$ & $1.1 \times 10^{-3}$ \\
Relative rms bunch momentum & $1.4 \times 10^{-4}$ \\
spread $\left(d p / p_{0}\right)_{\text {rms }}$ & \\
rms bunch length & $0.45 \mathrm{~m}$ \\
\hline \hline
\end{tabular}

use 8 families of arc chromatic sextupoles to correct the global second order chromaticities [26]. In this article, second order chromaticities are defined as $\xi_{x, y}^{(2)}=$ $\frac{1}{2}\left[\left(d^{2} Q_{x, y}\right) /\left(d \delta^{2}\right)\right]$, where $\delta=d p / p_{0}$. For this lattice, the second order chromaticities are about 2800 without correction. With correction, they are below 500 .

\section{Compensation strength}

In Sec. II, we discussed the head-on beam-beam compensation scheme with one beam-beam interaction and one e-lens. In RHIC, there are two proton-proton beam-beam interaction points at IP6 and IP8 and one e-lens close to IP10. According to Eq. (6), the total linear incoherent beam-beam tune shift with two collisions is

$$
\xi_{2 \mathrm{IP}}=\xi_{1 \mathrm{IP}} \times 2=-\frac{N_{p} r_{0} \beta_{\mathrm{IP}}^{*}}{4 \pi \gamma \sigma_{p, \mathrm{IP}}^{2}} \times 2=-\frac{N_{p} r_{0}}{2 \pi \epsilon_{n}} .
$$

Here $\xi_{1 I P}$ is the linear incoherent beam-beam tune shift from one collision, $\epsilon_{n}$ is the normalized transverse rms emittance of the proton beam. From Eq. (18), the total linear incoherent beam-beam tune shift is about -0.015 and -0.03 for bunch intensities $1.5 \times 10^{11}$ and $3.0 \times 10^{11}$, respectively.

We define the head-on beam-beam compensation strength as the fraction of the linear incoherent beambeam tune shift that is compensated. According to Eqs. (7) and (18), the compensation strength $\kappa$ is

$$
\kappa=\left(N_{e}^{*} / 2 N_{p}\right) /\left(\sigma_{e} / \sigma_{p, \text { e-lens }}\right)^{2} .
$$

$\sigma_{e}$ and $\sigma_{p \text {,e-lens }}$ are the transverse rms electron beam size and the transverse rms proton bunch size at the e-lens.

For simplicity, we define that half and full beam-beam compensation compensate the half and full total linear incoherent beam-beam tune shift. Their compensation strengths are 0.5 and 1 , respectively. If the electron beam has the same transverse rms beam size as the proton beam at the e-lens, that is, $\sigma_{e}=\sigma_{p \text {,e-lens }}$, we have $N_{e}^{*}=N_{p}$ and $N_{e}^{*}=2 N_{p}$ for half and full beam-beam compensation, respectively. In the following, except in the scan of the electron beam size, the electron beam always has the same transverse rms beam size as the proton beam at the e-lens.

\section{Phase advances between IPs and e-lens}

As discussed in Sec. II, we can adjust the betatron phase advances between the IPs and the e-lens to compensate the beam-beam resonance driving terms. For RHIC, all the arc focusing and defocusing quadrupoles are currently on the same power supply circuits. All the inner arcs and the outer arcs have almost the same phase advances. We only could slightly change the phase advances between IPs with the gamma-T jump quadrupoles. The gamma-T jump quadrupoles are used for the transition in the RHIC heavy ion runs. 
To cancel the nonlinear beam-beam tune shift and resonance driving terms introduced from IP8 with the e-lens at IP10, we decided to add two shunt power supplies to the main quadrupoles in the arc to allow us to change the phase advances between them $[27,28]$. Without adjustment, the horizontal and vertical phase advances between them are $(8.5 \pi, 11.1 \pi)$. In this simulation study, we set the phase advances between IP8 and the e-lens to be $(9 \pi, 11 \pi)$.

In the case of half beam-beam compensation, with the phase advances $(9 \pi, 11 \pi)$ between IP8 and the e-lens, the e-lens will completely compensate the beam-beam tune shift and beam-beam resonance driving terms from IP8 to the first order of linear incoherent beam-beam tune shift. In operations, the beam lifetime with collision is mainly determined by the beam-beam nonlinearity. We observed that the beam lifetime of bunches with one collision is about double the beam lifetime of bunches with two collisions.

For full beam-beam compensation, to compensate all the linear incoherent beam-beam tune shift and the beambeam resonance driving terms from IP6 and IP8 with one e-lens, the phase advances between IP6 to the e-lens also need to be odd multiples of $\pi$. This will require more independent arc quadrupole power supplies. In our current design, for minimum changes in hardware and lattices, we focus on half beam-beam compensation and the $k \pi$ phase advance adjustment between IP8 and the e-lens.

Following the procedure outlined in Sec. II, we numerically calculate the $2 \mathrm{D}$ beam-beam resonance driving terms and resonance widths. As an example, Figs. 2 and 3 show the horizontal 10th order beam-beam resonance driving terms and its resonance widths without beam-beam compensation ("No BBC"), with half beam-beam compensation ("Half BBC"), full beam-beam compensation

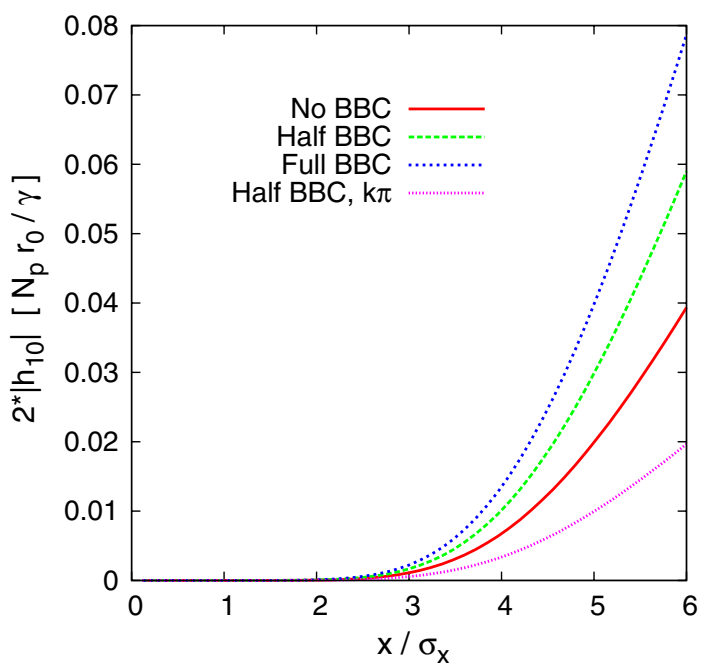

FIG. 2. 10th order horizontal beam-beam resonance driving terms under different beam-beam conditions. The horizontal axis is the particle's amplitude in units of $\sigma_{x}$. The vertical axis is the resonance driving term in units of $N_{p} r_{0} / \gamma$.

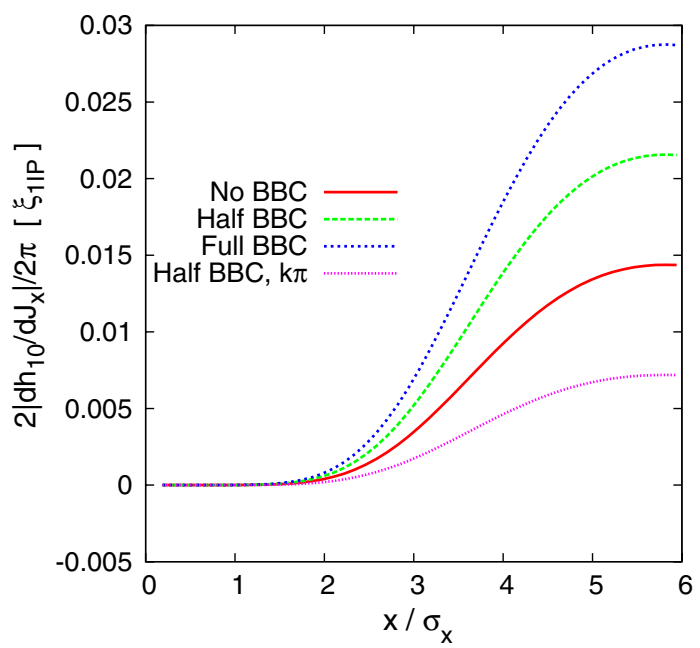

FIG. 3. 10th order horizontal beam-beam resonance widths under different beam-beam conditions. The vertical axis is in units of the incoherent beam-beam tune shift $\xi_{1 \mathrm{IP}}$ with one IP.

("Full BBC"), and half beam-beam compensation with the phase advances $(9 \pi, 11 \pi)$ between IP8 and the e-lens ("Half BBC, $k \pi$ ").

For the 10th order beam-beam resonance, half beambeam compensation without phase advance adjustment gives larger beam-beam resonance driving terms than without compensation. Full beam-beam compensation gives the largest beam-beam resonance driving terms. Half beam-beam compensation with phase advances $(9 \pi, 11 \pi)$ between IP8 and the e-lens compensates about half of the beam-beam resonance driving term and reduces the beam-beam resonance width by about half. We also calculated the beam-beam resonance driving terms and resonance widths for other orders. We found that, for the most of orders, full beam-beam compensation gives the largest beam-beam resonance driving terms.

\section{E. Simulation model}

In the following simulation study, we track the proton particles element by element [29]. The nonlinear magnetic field errors in the triplets and separation dipoles in the interaction regions are included. Each magnetic element is modeled with a 6D symplectic transfer map. We adopted the 4th order symplectic integration by Ruth [30]. To save time in the long-term particle tracking, we model the magnetic multipoles as thin lens kicks. Tunes and chromaticities are rematched before tracking.

Considering that $\beta^{*}$ is comparable to the proton bunch length, we use the 6D weak-strong synchrobeam map $[31,32]$ to model the proton-proton beam-beam interaction at IP6 and IP8. In the code, the strong bunch is split into 11 slices.

In our case, since the e-lens is working in a DC mode, its electric and magnetic fields are static. In the code, we split the $2 \mathrm{~m}$ long e-lens into 8 slices. Each slice is modeled as a 
drift-a 4D weak-strong beam-beam kick-a drift. The 4D weak-strong beam-beam kick is given by Bassetti and Erskine [33].

To fully use the available tune space between $2 / 3$ and $7 / 10$ and for better comparison of the simulation results under different beam-beam conditions, we fix the zeroamplitude particle tunes at $(0.67,0.68)$ under different beam-beam conditions, except in the proton working point scan. The RHIC polarized proton operation experience shows that a lower working point between $2 / 3$ and $7 / 10$ is preferable to obtain a better beam-beam lifetime and to preserve the proton polarization at store. In the simulation, the linear chromaticities are set to $(1,1)$.

\section{FREQUENCY MAP ANALYSIS}

In this section, we use frequency map analysis [34,35] to calculate the tune footprint and tune diffusion of the proton beam. We focus on three beam-beam conditions: without beam-beam compensation, with half and full head-on beam-beam compensation. Four proton bunch intensities are used: $1.5 \times 10^{11}, 2.0 \times 10^{11}, 2.5 \times 10^{11}$, and $3.0 \times 10^{11}$. For each beam-beam condition, we track single protons up to 2048 turns.
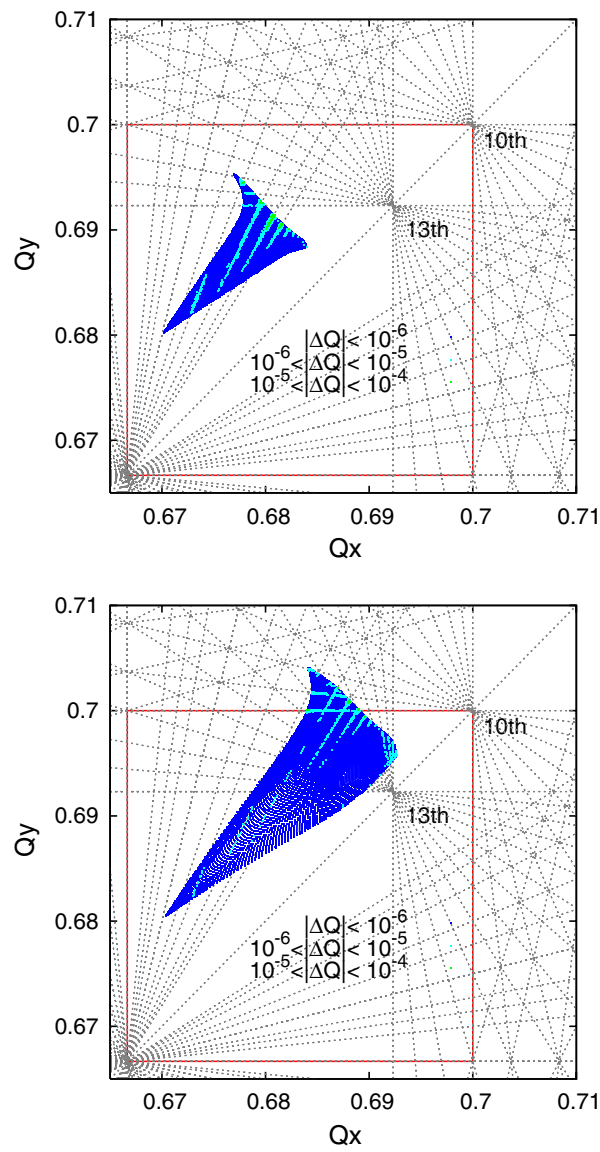

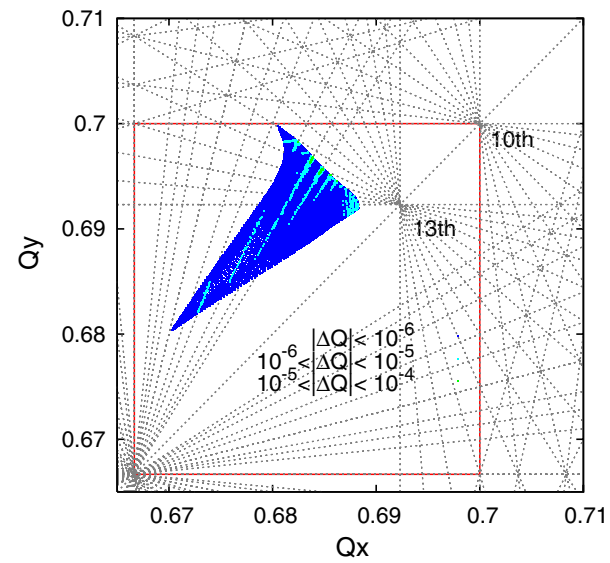

The area occupied by the beam in the tune space is called the tune footprint. In our case, with beam-beam interaction, the tune spread is determined by beam-beam. To quantify the stability of single particle motion, we measure the tune diffusion in the 2048 turns through the observation of

$$
|\Delta Q|=\sqrt{\left|\Delta Q_{x}\right|^{2}+\left|\Delta Q_{y}\right|^{2}},
$$

where $\left|\Delta Q_{x}\right|$ and $\left|\Delta Q_{y}\right|$ are the horizontal and vertical betatron tune differences between the first and second 1024 turns. Normally, the smaller the tune diffusion is, the more stable the particle motion.

Tune diffusion of particles can be plotted with different colors in the $\left(Q_{x}, Q_{y}\right)$ plane or in the $\left(x / \sigma_{x}, y / \sigma_{y}\right)$ plane $[36,37]$. In our convention, the deep blue dots mean that $|\Delta Q|<10^{-6}$. The light blue dots mean that $10^{-6}<$ $|\Delta Q|<10^{-5}$. The green and the yellow mean $10^{-5}<$ $|\Delta Q|<10^{-4}$ and $10^{-4}<|\Delta Q|<10^{-3}$, respectively. In the $\left(Q_{x}, Q_{y}\right)$ plane, the betatron resonance lines up to 13th order are plotted.

In this study, the initial coordinates of protons are uniformly sampled from 0 to $6 \sigma$ with a step size of $0.05 \sigma$ in the first quadrant of the $\left(x / \sigma_{x}, y / \sigma_{y}\right)$ plane. The initial

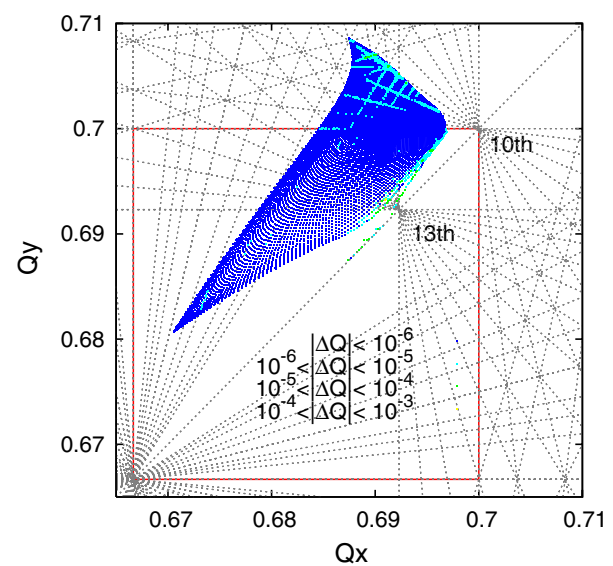

FIG. 4. Tune footprints and tune diffusion without beam-beam compensation. The proton beams collide at IP6 and IP8. The bunch intensities are $1.5 \times 10^{11}$ (top left), $2.0 \times 10^{11}$ (top right), $2.5 \times 10^{11}$ (bottom left), and $3.0 \times 10^{11}$ (bottom right). 
conjugate momenta are set to zero. The betatron tunes of single particle motion are determined with an interpolated fast-Fourier transform and a Hanning window [38]. To avoid tune modulation due to synchrotron motion, the rf is turned off in this section. In the following, we only provide the frequency map analysis for on-momentum particles. The footprint of off-momentum particles is slightly shifted in the tune space by the off-momentum tune shifts. The tune diffusion for the off-momentum particles is different from that of on-momentum particles.

\section{A. Without compensation}

Figure 4 shows the tune footprints and tune diffusion only with beam-beam interaction at IP6 and IP8 only. With the zero-amplitude particle tunes fixed at $(0.67,0.68)$, the tunes of large amplitude particles are higher than the zeroamplitude tunes. With the increase of the proton bunch intensity, the tune spread gets bigger and the footprint stretches up to the 13th order resonance lines crossing $(9 / 13,9 / 13)$ and even to the 10th order resonance lines crossing $(7 / 10,7 / 10)$. From the simulation calculation, the beam-beam tune spreads from 0 to $6 \sigma$ are $0.016,0.020$, 0.024 , and 0.028 for the proton bunch intensities $1.5 \times 10^{11}$, $2.0 \times 10^{11}, 2.5 \times 10^{11}$, and $3.0 \times 10^{11}$, respectively. The
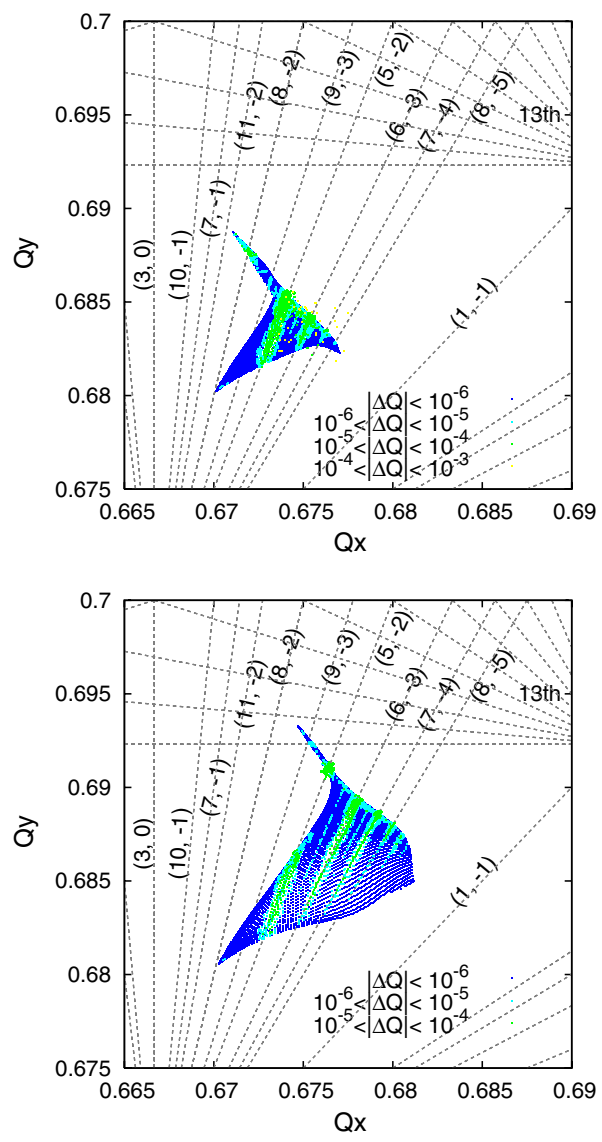

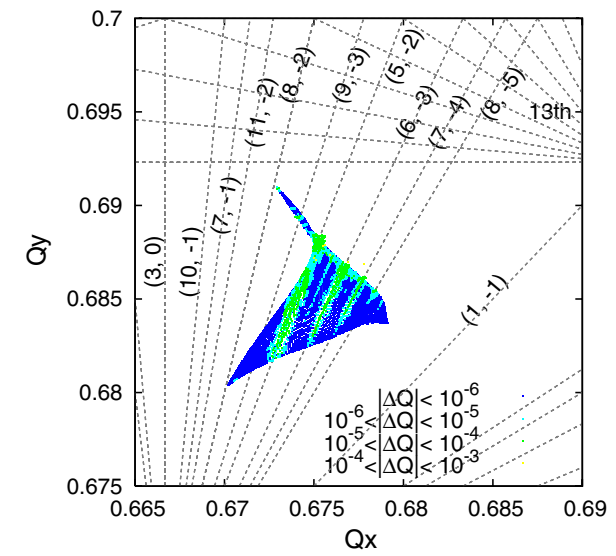

beam-beam tune spreads are close to the total linear incoherent beam-beam tune shifts given by Eq. (18).

The figure of merit of the RHIC polarized proton operation with longitudinally polarized beams is $L P^{4}$, where $L$ is the luminosity and $P$ is the polarization of the proton beams. From Fig. 4, when the bunch intensity is bigger than $2.0 \times 10^{11}$, the tune footprint up to $6 \sigma$ will touch or cross $7 / 10$. 7/10 is not only a 10 th order betatron resonance line but also a spin depolarization resonance line. Experiments and simulations have shown that the beambeam lifetime and the proton polarization are reduced when the vertical tune of the proton beam is close to $7 / 10$. With an upgraded polarized proton source, the proton bunch intensity in RHIC will reach $3.0 \times 10^{11}$. To reduce the large beam-beam tune spread and to compensate the large beam-beam resonance driving terms with such a high proton bunch intensity, we planned to install head-on beam-beam compensation in RHIC.

\section{B. Half compensation}

Figure 5 shows the tune footprints and tune diffusion with half head-on beam-beam compensation. Compared to Fig. 4, the tune footprints are squeezed towards the zeroamplitude particle tunes $(0.67,0.68)$. For the same proton

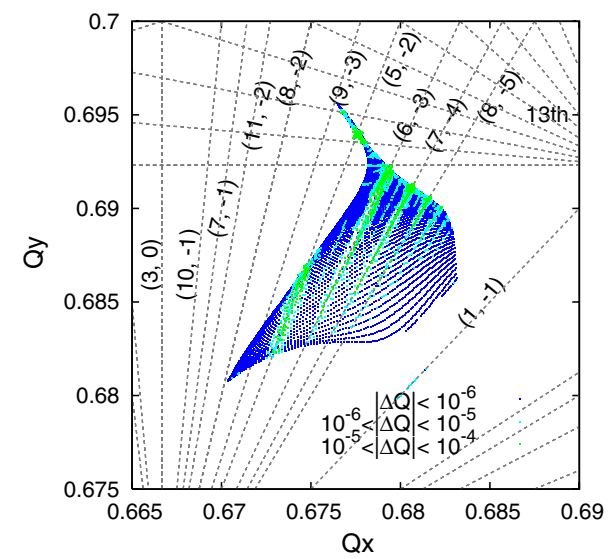

FIG. 5. Tune footprints and tune diffusion with half head-on beam-beam compensation. The bunch intensities are $1.5 \times 10^{11}$ (top left), $2.0 \times 10^{11}$ (top right), $2.5 \times 10^{11}$ (bottom left), and $3.0 \times 10^{11}$ (bottom right). 

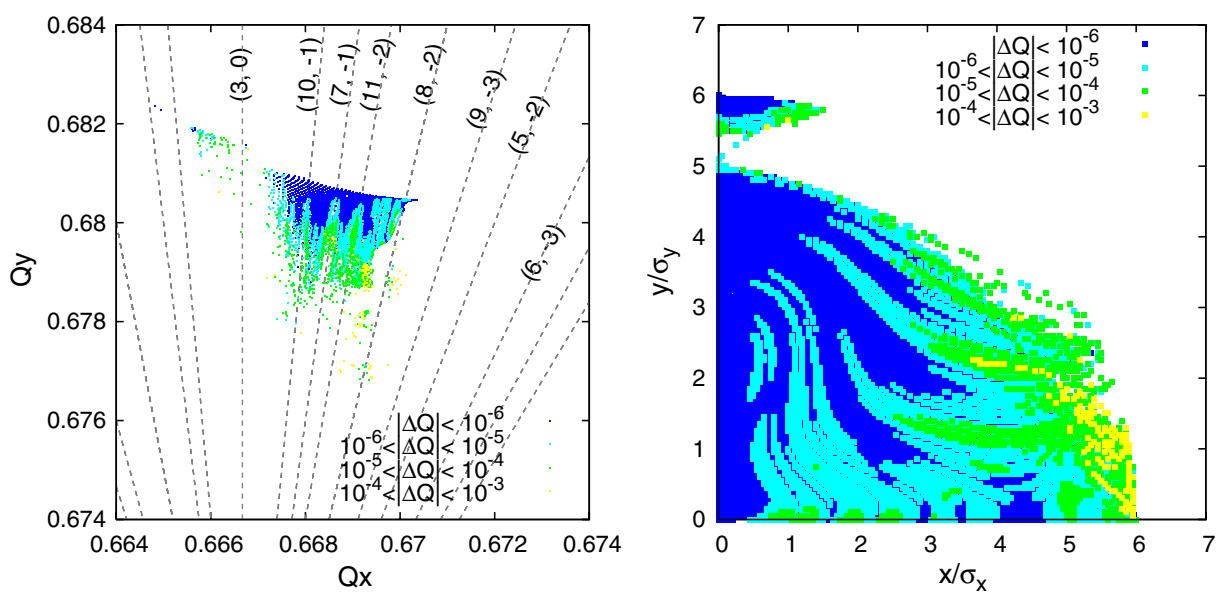

FIG. 6. Tune footprint (left) and tune diffusion map (right) of full head-on beam-beam compensation with the proton bunch intensity $2.5 \times 10^{11}$. The fractional zero-amplitude tunes are $(0.67,0.68)$. Particle loss is observed in 2048 turns.

bunch intensity, the beam-beam tune spread is reduced roughly by half. Even with the bunch intensities $2.5 \times 10^{11}$ and $3.0 \times 10^{11}$, their tune footprints with half head-on beam-beam compensation are well below the resonance line $Q_{y}=7 / 10$.

In Fig. 5, some resonance lines, such as $(5,-2)$, $(6,-3),(7,-4)$, and $(8,-5)$, can be clearly identified with tune diffusion. They do not necessarily cause direct particle loss since they are difference resonances. Foldings in the tune footprints [39] are observed at larger particle amplitudes. They are caused by the different signs of the detunings from the beam-beam interaction and the magnetic nonlinearity in the lattice. With half beam-beam compensation, the most important resonances in the tune space are the horizontal third order resonance $3 Q_{x}$ and the 13 th order resonances. Both of them are not excited by the beam-beam interaction and beam-beam compensation. However, according to Refs. [16,21], high order beambeam resonances still can cause resonance overlapping and chaotic motion even in a low order resonance free tune space.

\section{Full compensation}

With full beam-beam compensation, the size of the tune footprint is greatly reduced and is comparable to that without any beam-beam interaction. As an example, Fig. 6 shows the tune footprint (left) and the tune diffusion map (right) with the bunch intensity $2.5 \times 10^{11}$ in the $\left(Q_{x}, Q_{y}\right)$ and $\left(x / \sigma_{x}, y / \sigma_{y}\right)$ planes. The right plot shows that there are particles lost in 2048 turns.

With full beam-beam compensation, the large amplitude particles have lower tunes than the zero-amplitude tunes. With the zero-amplitude tunes fixed at $(0.67,0.68)$, the horizontal tunes of large amplitude particles are close to or even cross the horizontal 3 rd order betatron resonance at $Q_{x}=2 / 3$. The width of $Q_{x}=2 / 3$ is given by the magnetic nonlinearity in the lattice. Experimentally, without any beam-beam interaction, we measured the stop band of this resonance about 0.005 [40].

Since the beam-beam tune spread is largely reduced with half and full head-on beam-beam compensation, it is possible to scan the proton working point in the tune space between $2 / 3$ and $7 / 10$ to search for a good working point with a better beam lifetime.

\section{Summary}

From the above frequency map analysis, we conclude that there is not enough tune space between $2 / 3$ and $7 / 10$ to hold the large beam-beam tune spread when the proton bunch intensity is higher than $2.0 \times 10^{11}$. Head-on beambeam compensation is very effective to reduce the beambeam tune spread and is needed for high proton bunch intensities. However, considering that the e-lens is a nonlinear element, direct long-term particle tracking is needed to determine the effects of head-on beam-beam compensation on the proton dynamic aperture and beam lifetime.

\section{DYNAMIC APERTURE CALCULATION}

In this section, we show calculations of the proton dynamic aperture with head-on beam-beam compensation in RHIC. The dynamic aperture is defined as the maximum betatron amplitude within which particles are not lost after a certain number of tracking turns [41]. The long-term dynamic aperture converges to the boundary between regular and chaotic motion [42].

For a lower proton bunch intensity, the dynamic aperture is mainly decided by the magnetic nonlinearity. For a higher proton bunch intensity, the dynamic aperture will be reduced by beam-beam interaction. With head-on beambeam compensation, the proton particle's dynamic aperture is determined by the beam-beam interaction and beam-beam compensation, the nonlinear magnetic fields in the lattice, and the working point choosing. 
We will track single proton particles up to $10^{6}$ turns. The zero-amplitude particle tunes are set to $(28.67,29.68)$ under all beam-beam conditions except during the tune scan. The linear chromaticities are corrected to $(1,1)$ before tracking. For each beam-beam condition, we search the dynamic aperture in 10 equally spaced angles in the first quadrant in the $\left(x / \sigma_{x}, y / \sigma_{y}\right)$ plane. We focus on comparing the minimum dynamic aperture among the 10 angles. The dynamic aperture is given in units of rms transverse beam size $\sigma$.

In this study, the $\mathrm{rf}$ is turned on. The relative $\mathrm{rf}$ momentum acceptance is $\left(d p / p_{0}\right)_{\max }=1.1 \times 10^{-3}$. The initial relative off-momentum deviation is set to 0.00042 , which is 3 times of the rms relative momentum spread of the proton beam. For comparison, we also used smaller or larger initial relative off-momentum deviation for some cases. The tracking results with different initial off-momentum deviation will not affect our conclusions.

\section{A. Without and with compensation}

First, we calculate the dynamic apertures without, with half, and full head-on beam-beam compensation. The proton bunch intensity varies from $1.0 \times 10^{11}$ to $3.0 \times 10^{11}$. In this calculation, the betatron phase advances between IP8 and the e-lens are the default ones $(8.5 \pi, 11.1 \pi)$. Figure 7 shows the results.

Without beam-beam compensation, the dynamic aperture declines with increasing proton bunch intensity. With a higher proton bunch intensity, the tune footprint and tune spread get bigger. With the zero-amplitude tunes fixed at $(0.67,0.68)$, the tunes of large amplitude particles move up to the 13th and even 10th resonances. Another reason is that the beam-beam resonance driving terms and resonance widths increase with the bunch intensity.

Compared to the case without beam-beam compensation, half head-on beam-beam compensation improves the

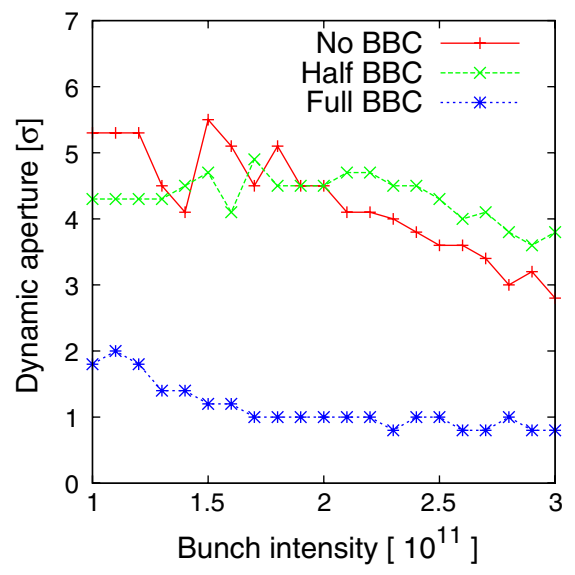

FIG. 7. Dynamic apertures without beam-beam compensation and with half and full beam-beam compensation. The proton bunch intensity ranges from $1.0 \times 10^{11}$ to $3.0 \times 10^{11}$. The phase advance adjustment between IP8 and the e-lens is not included. dynamic aperture for a proton bunch intensity above $2.0 \times$ $10^{11}$. With half beam-beam compensation, the beam-beam tune spread of a higher bunch intensity is reduced and the tune footprint is located below the resonance line $Q_{y}=$ $7 / 10$. For a proton bunch intensity less than $2 \times 10^{11}$, half compensation does not increase the dynamic aperture. This may be explained by the fact that half beam-beam compensation with the default phase advances between IP8 and the e-lens increases the beam-beam resonance driving terms and resonance widths as shown in Figs. 2 and 3.

From Fig. 7, full beam-beam compensation gives the smallest dynamic apertures. With full beam-beam compensation, the beam-beam tune spread is largely compensated. With the zero-amplitude tunes fixed at $(0.67,0.68)$, the horizontal tunes of large amplitude particles are very close to the 3rd order betatron resonance line $Q x=2 / 3$. Even without any beam-beam interaction, with the zeroamplitude tunes fixed at $(0.67,0.68)$, the dynamic aperture is only $3 \sigma$. Another reason for the small dynamic aperture is that full beam-beam compensation generates the largest beam-beam resonance driving terms and widest beambeam resonance widths.

\section{B. Compensation strength}

Here we scan the compensation strength of head-on beam-beam compensation. In this study, we assume that the electron beam size is the same as the proton bunch beam size at the e-lens. We adjust the electron beam intensity to change the beam-beam compensation strength. The betatron phase advances between IP8 and the e-lens are not optimized here. Figure 8 shows the results.

From Fig. 8, for all of the four proton bunch intensities, the dynamic apertures begin to drop sharply when the compensation strength exceeds 0.7 . For the bunch intensities $2.5 \times 10^{11}$ and $3.0 \times 10^{11}$, head-on beam-beam compensation with a compensation strength less than 0.6

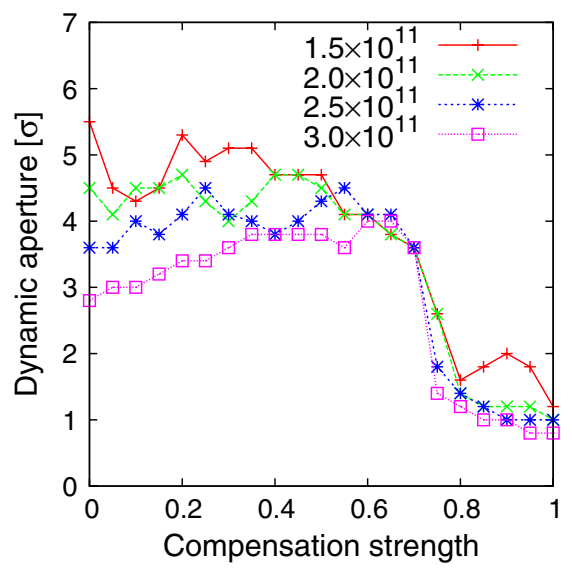

FIG. 8. Dynamic apertures of four proton bunch intensities versus head-on beam-beam compensation strength. For half and full beam-beam compensation, the compensation strengths are 0.5 and 1.0, respectively. 
improves the proton beam dynamic aperture. The peak dynamic apertures occur at the compensation strength around 0.5-0.6. The peak dynamic apertures are about $1.0 \sigma$ higher than that without beam-beam compensation.

\section{Half and full compensations}

Here we calculate the dynamic apertures of half and full beam-beam compensation with the betatron phase advance adjustment between IP8 and the e-lens. Before adjustment, the phase advances between them are $(8.5 \pi, 11.1 \pi)$. With adjustment, they are $(9 \pi, 11 \pi)$. We also apply second order chromaticity correction on top of the phase advance adjustment. Before correction, the second order chromaticities are around 2800. With correction, they are below 500.

Figure 9 shows the dynamic aperture of half beam-beam compensation with the phase advance adjustment. The proton bunch intensity is varied from $1.0 \times 10^{11}$ to $3.0 \times 10^{11}$. With the phase advance adjustment, the dynamic aperture of half beam-beam compensation is improved for all bunch intensities. Even in the lower proton bunch intensity range, the dynamic aperture of half headon beam-beam compensation with the phase adjustment is better or comparable to that without compensation. The reason for this improvement is that the beam-beam resonance driving terms and resonance widths are better compensated between IP8 and the e-lens with $(9 \pi, 11 \pi)$ phase advances between them.

Figure 10 shows the dynamic aperture of full beambeam compensation with the above phase advance adjustment between IP8 and the e-lens. The phase advance adjustment improves the dynamic aperture too, especially for the lower proton bunch intensities. However, even with the phase advance adjustment, the dynamic aperture of full beam-beam compensation is still much smaller than that without compensation. The main reason is that the working point is close to the $Q_{x}=2 / 3$ resonance line. The dynamic aperture versus the working point will be studied later.

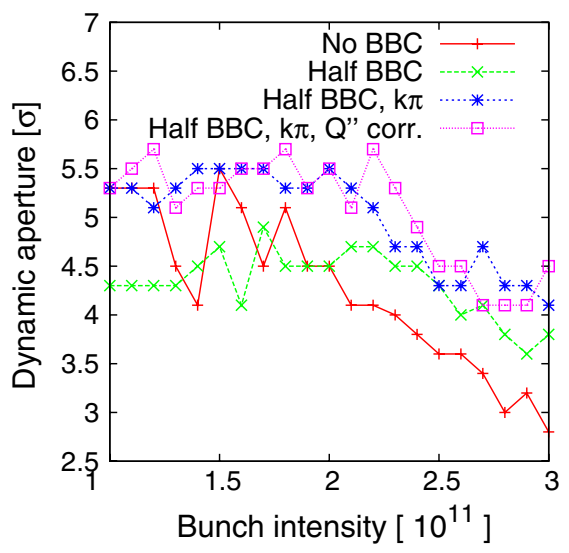

FIG. 9. Dynamic apertures of half head-on beam-beam compensation with the betatron phase advance adjustment and the global second order chromaticity correction.

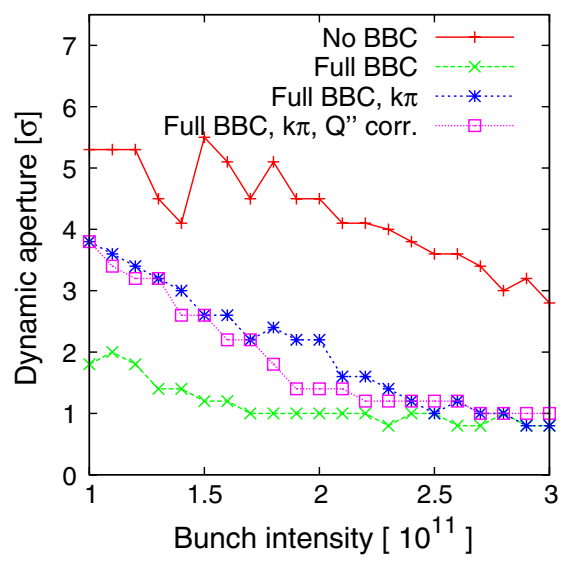

FIG. 10. Dynamic apertures of full head-on beam-beam compensation with the betatron phase advance adjustment and the global second order chromaticity correction.

Figures 9 and 10 also show the dynamic aperture with second order chromaticity correction. The goal of second order chromaticity correction is to reduce the offmomentum tune shift and the off-momentum $\beta$ beat. In our current second order chromaticity correction scheme, we do not optimize the sextupole resonance driving terms at the same time. From Fig. 9, the second order chromaticity correction further improves the dynamic aperture of half beam-beam compensation for a proton bunch intensity between $2.0 \times 10^{11}$ and $2.5 \times 10^{11}$, but reduces the dynamic aperture for a proton bunch intensity between $2.5 \times 10^{11}$ and $3.0 \times 10^{11}$.

\section{Proton working point}

In the above calculation, the zero-amplitude tunes of the proton beam are fixed at $(0.67,0.68)$. With beam-beam compensation, the tune footprint becomes smaller and it is

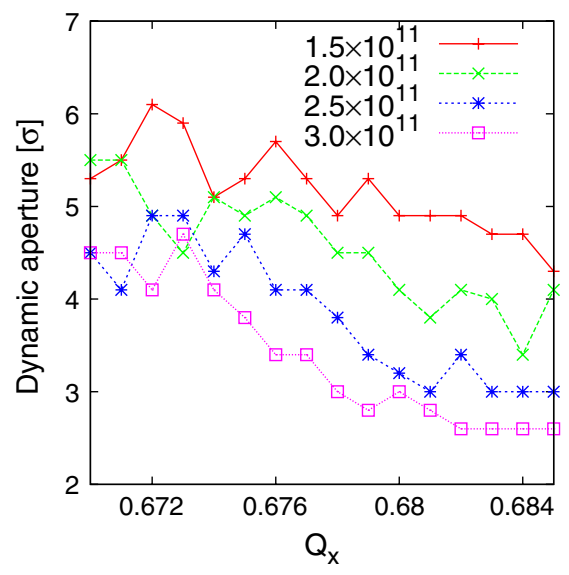

FIG. 11. Dynamic apertures of half head-on beam-beam compensation in the scan of proton working point. The horizontal axis is the fractional horizontal zero-amplitude tune. The fractional vertical zero-amplitude tune is always 0.01 higher than the horizontal one. 


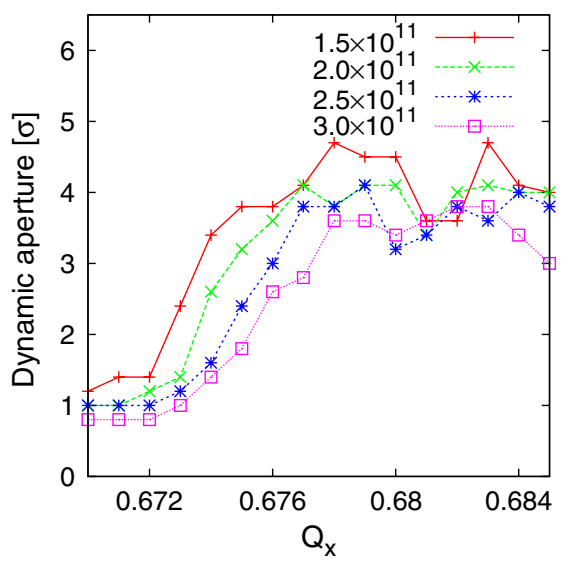

FIG. 12. Dynamic apertures of full head-on beam-beam compensation in the scan of proton working point. The horizontal axis is the fractional horizontal zero-amplitude tune. The fractional vertical zero-amplitude tune is always 0.01 higher than the horizontal one.

possible to scan the proton working point between $2 / 3$ and $7 / 10$ to search for a better working point to maximize the dynamic aperture. In this study, the phase advance adjustment between IP8 and the e-lens are included.

Figure 11 shows the dynamic aperture of half beambeam compensation in the tune scan. The horizontal axis is the horizontal zero-amplitude tune. The vertical zeroamplitude tune is always 0.01 higher than the horizontal one. From Fig. 11, the dynamic aperture decreases for all the four bunch intensities when the tunes are moved up from $(0.67,0.68)$ to $(0.685,0.695)$. The reason is that the tune footprint with higher zero-amplitude tunes will move up to the 10th order resonance lines.

Figure 12 shows the dynamic aperture of full beambeam compensation in the tune scan. The dynamic aperture increases when the zero-amplitude tunes are moved up from $(0.67,0.68)$ to $(0.685,0.695)$. The reason is that the tune footprint with higher zero-amplitude tunes moves away from the 3 rd order resonance line $Q_{x}=2 / 3$.

Comparing Fig. 11 with Fig. 12, we find that for proton bunch intensity from 1.5 to $3.0 \times 10^{11}$ and the shown tune range, a larger dynamic aperture can be reached with half beam-beam compensation than with full beam compensation.

\section{E. Mismatched electron beam size}

In the above calculation, we assume that the electron beam always has the same transverse rms beam size as the proton bunch at the e-lens. In the following, we first vary the electron beam size from $40 \%$ to $160 \%$ of the proton beam size for half beam-beam compensation. In this study we keep the electron bunch intensity constant. Therefore, the beam-beam compensation strength will be changed when we change the electron beam size. The zeroamplitude tunes are always rematched to $(0.67,0.68)$ before tracking.

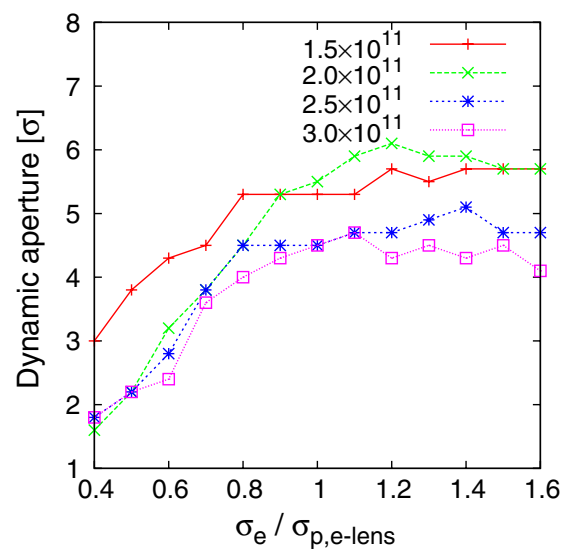

FIG. 13. Dynamic aperture of half head-on beam-beam compensation with a mismatched electron beam size. The electron bunch intensity is keep constant here. Four proton bunch intensities are used.

Figure 13 shows the results. The dynamic aperture drops for all the four bunch intensities when the electron beam size is $20 \%$ smaller than that of the proton bunch. With an electron beam size smaller than that of the proton bunch, the beam-beam compensation strength is increased. On the other hand, if the electron beam size is larger than that of the proton bunch, the beam-beam compensation strength is reduced and the beam-beam tune spread is not effectively compensated, although the dynamic aperture does not decrease.

Figure 14 shows the dynamic aperture in a $2 \mathrm{D}$ scan of the electron beam intensity and its beam size. The proton bunch intensity is $2.5 \times 10^{11}$. The zero-amplitude tunes are fixed at $(0.67,0.68)$. In this plot, the red and the blue curves represent full and half beam-beam compensation. Full and more than full beam-beam compensation gives dynamic aperture less than $2 \sigma$. The area with a larger dynamic aperture is around half beam-beam compensation with

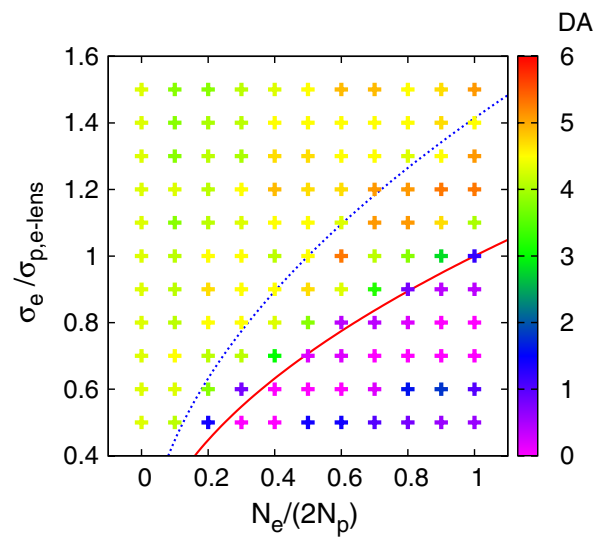

FIG. 14. Dynamic apertures in a 2D scan of the electron beam intensity and beam size. The proton bunch intensity is $2.5 \times 10^{11}$. The blue and red curves represent half and full beam-beam compensation, respectively. 
the electron beam size slightly larger than the proton beam. To keep the same compensation strength, according to Eq. (19), we have to increase the electron beam intensity quadratically when we increase the electron beam size. Therefore, the optimum electron beam size for half beam-beam compensation ranges from 1 to $1.2 \sigma_{p \text {,e-lens }}$.

\section{F. Summary}

With the zero-amplitude tunes fixed at $(0.67,0.68)$, we found that half head-on beam-beam compensation improves the proton dynamic aperture and full head-on beam-beam reduces the dynamic aperture. The optimal compensation strengths for the proton bunch intensities $2.5 \times 10^{11}$ and $3.0 \times 10^{11}$ are around 0.5-0.6. The $k \pi$ betatron phase advances between IP8 and the e-lens further improve the dynamic aperture of half beam-beam compensation. Tune scans show that half head-on beam-beam with a lower working point and full head-on beam-beam compensation with a higher working point give larger dynamic apertures. The scan of the electron beam intensity and its beam size shows that the optimum compensation is around half beam-beam compensation with the electron beam size slightly larger than the proton beam size.

\section{BEAM LOSS RATE CALCULATION}

In this section, we calculate the proton beam loss rate with multiparticle tracking in the presence of head-on beam-beam compensation. Different from the above dynamic aperture calculation, the initial coordinates of macroparticles are sampled from a 6D Gaussian distribution. Limited by computing capacity, in most cases we track 4800 macroparticles up to $2 \times 10^{6}$ turns. $2 \times 10^{6}$ turns are 24 seconds.

\section{A. Simulation setup}

The initial coordinates of macroparticles are generated with a Gaussian distribution generator [43]. Particles with large transverse amplitudes and large momentum deviations are likely lost in long-term tracking. However, for a limited number of macroparticles sampled from a solid Gaussian distribution, there are only a few macroparticles in the Gaussian bunch tail. To detect a small beam loss in $2 \times 10^{6}$ turns without increasing the number of macroparticles, we track particles with an initial hollow Gaussian distribution [44-48].

In this approach, we assume that the particles in the bunch core are stable and will not be lost in $2 \times 10^{6}$ turns. To save computing time, we only track macroparticles whose transverse or longitudinal amplitudes are bigger than a certain rms beam size. The boundary between the stable core and the unstable bunch tail is carefully chosen. We first calculate the dynamic aperture and set the boundary well below it. After tracking, we will check if there are lost particles whose initial amplitudes are close to the boundary. If so, we must lower the boundary and track again.

The amplitude distribution of the particles in a $6 \mathrm{D}$ Gaussian bunch is

$$
\rho\left(n_{x}, n_{y}, n_{z}\right)=n_{x} n_{y} n_{z} e^{-\left(n_{x}^{2}+n_{y}^{2}+n_{z}^{2}\right) / 2},
$$

where $n_{x, y, z}$ are the horizontal, vertical, and longitudinal amplitudes in units of $\sigma . n_{x, y, z}^{2}=2 J_{x, y, z} / \epsilon_{\mathrm{rms}, x, y, z}$, where $J_{x, y, z}$ and $\epsilon_{\mathrm{rms}, x, y, z}$ are the particle's actions and the beam's rms emittances. For a hollow 6D Gaussian distribution, if we choose the transverse boundary $n_{t} \sigma$, where $n_{t}=$ $\sqrt{n_{x}^{2}+n_{y}^{2}}$, and the longitudinal boundary $n_{l} \sigma$, the fraction of the particles to be tracked is

$$
\begin{aligned}
P= & 1-\int_{0}^{n_{t}} \int_{0}^{\sqrt{n_{t}^{2}-n_{y}^{2}}} n_{x} n_{y} e^{-\left(n_{x}^{2}+n_{y}^{2}\right) / 2} d n_{x} d n_{y} \\
& \times \int_{0}^{n_{l}} n_{z} e^{-n_{z}^{2} / 2} d n_{z} .
\end{aligned}
$$

For example, if we choose $n_{t}=3.5$ and $n_{l}=3.5,4800$ macroparticles of the hollow distribution represent 270586 macroparticles of a solid 6D Gaussian distribution.

\section{B. An example}

Here we give an example of the proton beam loss rate calculation with an initial hollow Gaussian distribution. The proton bunch intensity is $2.5 \times 10^{11}$ and half headon beam-beam compensation is included. If we track with 4800 macroparticles of a solid 6D Gaussian distribution, there is only 1 macroparticle lost in $2 \times 10^{6}$ turns. The calculated beam loss rate is $3.1 \%$ /hour. For comparison, we track only macroparticles whose transverse amplitude or longitudinal amplitude is above $3.5 \sigma$.

Figure 15 shows the initial transverse amplitudes of the 4800 macroparticles with red dots. There are some macroparticles below transverse amplitudes of $3.5 \sigma$ because their longitudinal amplitudes are larger than $3.5 \sigma$ so that they will be tracked too. In the simulation, we set the physical aperture to $6 \sigma$ which is close to the RHIC actual collimation settings. After $2 \times 10^{6}$ turns, 17 out of 4800 macroparticles are lost in the example. In Fig. 15, the initial transverse amplitudes of the lost macroparticles are shown in blue. All the lost macroparticles have initial transverse amplitudes above $3.5 \sigma$.

Since the 4800 macroparticles represent 270586 macroparticles of a solid 6D Gaussian distribution proton bunch, the percentage of particle loss of the proton bunch in $2 \times 10^{6}$ turns is $17 / 270586=0.006 \%$. The proton beam loss rate is $0.97 \%$ /hour. For the same beam-beam condition, Fig. 16 shows the particle loss in $2 \times 10^{6}$ turns with 5 different sets of initial macroparticle coordinates. The vertical axis is the relative intensity of the proton beam. The calculated beam loss rate from the 5 cases is $(1.1 \% \pm 0.2 \%) /$ hour. 


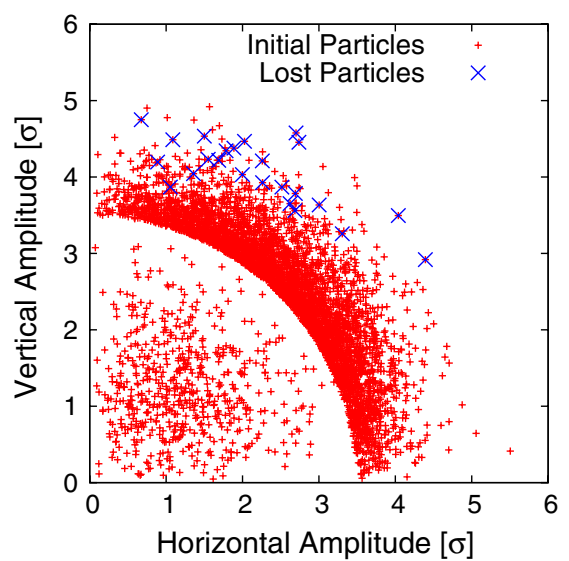

FIG. 15. Initial coordinates of 4800 tracked particles (in red) and of the lost particles (in blue). In this example, the proton bunch intensity is $2.5 \times 10^{11}$. Half beam-beam compensation is used. These 4800 macroparticles represent 270586 macroparticles of a solid 6D Gaussian distribution. After $2 \times 10^{6}$ turns, 17 macroparticles are lost.

Tracking a hollow Gaussian distribution is efficient when the dynamic aperture is between $2.5 \sigma$ to $5 \sigma$. When the dynamic aperture is less than $2.5 \sigma$, we simply track with a solid 6D Gaussian distribution. When it is above $5 \sigma$, a special Gaussian tail generator may be needed [43]. The good store beam decay is a few percent per hour in the RHIC polarized proton operation with proton bunch intensities around $1.5 \times 10^{11}$. Currently, the calculated beam loss rate from our simulation is about 10 times smaller than the actual beam decay measurement in RHIC. To bridge the gap between the simulation and measurement, a more realistic magnetic lattice model together with the beam and parameter modulations or noise $[49,50]$ should be included in the simulation.

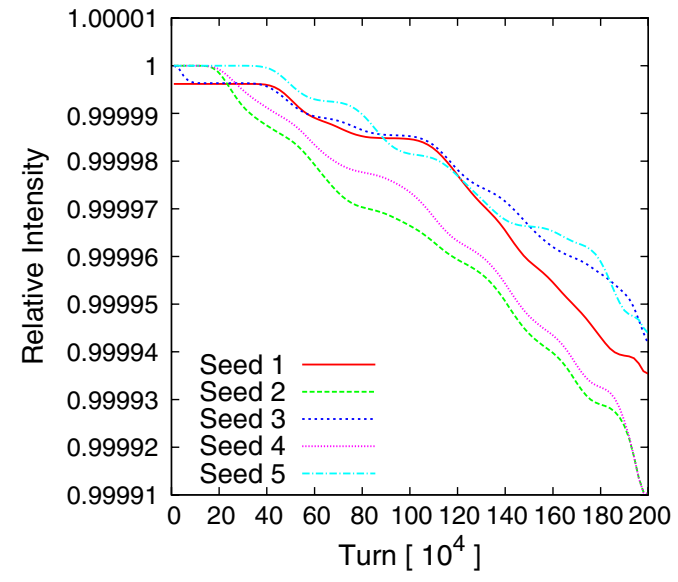

FIG. 16. Proton beam losses in $2 \times 10^{6}$ turns with 5 different sets of initial macroparticle coordinates. Proton bunch intensity is $2.5 \times 10^{11}$. Half beam-beam compensation is used. The calculated proton beam loss rate from the 5 cases is $(1.1 \% \pm$ $0.2 \%$ )/hour.

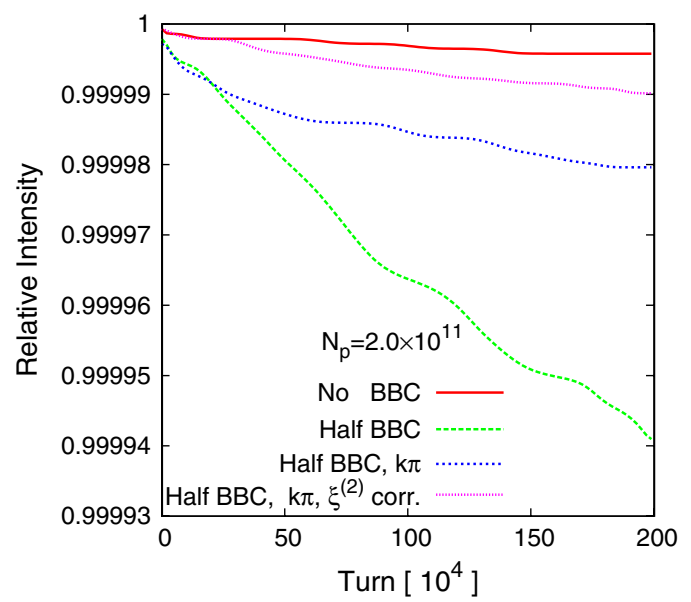

FIG. 17. Relative proton beam losses in $2 \times 10^{6}$ turns for the proton bunch intensity $2.0 \times 10^{11}$. The losses without beambeam compensation, with half beam-beam compensation, with the betatron phase advance adjustment, and with the global second order chromaticity correction $\left(\xi^{(2)}\right.$ corr.) are shown.

In the following, we will focus on comparing the relative beam losses in $2 \times 10^{6}$ turns under different beam-beam conditions. For each beam-beam condition, we normally track with different sets of initial particle distribution. For some condition, we double the number of macroparticles or the maximum number of tracking turns.

\section{Tracking results}

We first calculate the relative proton beam losses in $2 \times 10^{6}$ turns under different beam-beam compensation conditions. In this study, the zero-amplitude tunes of the proton beam are still set to $(0.67,0.68)$. The linear chromaticities are $(1,1)$. Three proton bunch intensities are

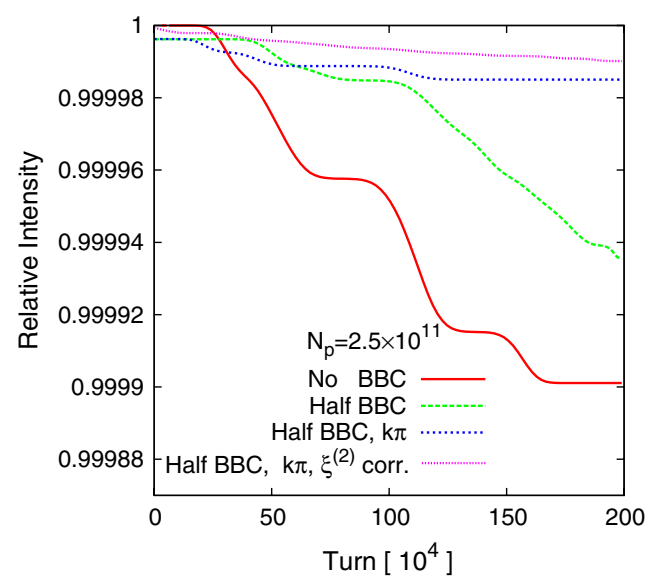

FIG. 18. Relative proton beam losses in $2 \times 10^{6}$ turns for the proton bunch intensity $2.5 \times 10^{11}$ without beam-beam compensation, with half beam-beam compensation, with the betatron phase advance adjustment, and with the global second order chromaticity correction. 


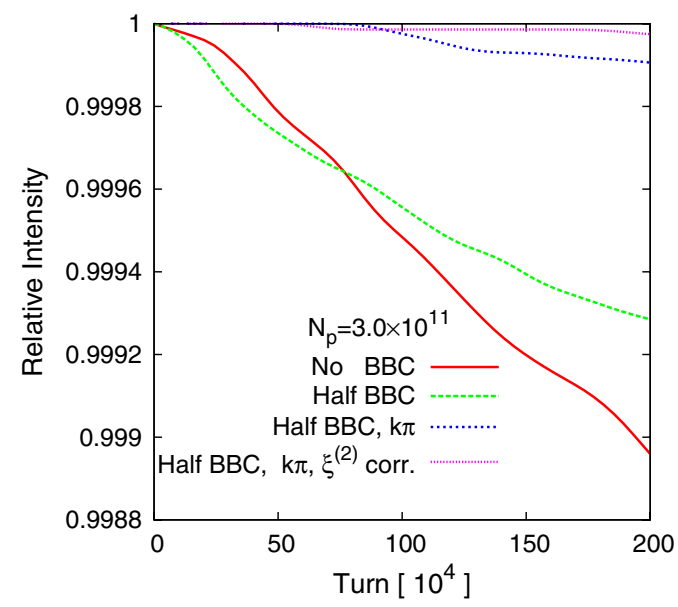

FIG. 19. Relative proton beam losses in $2 \times 10^{6}$ turns for the proton bunch intensity $3.0 \times 10^{11}$ without beam-beam compensation, with half beam-beam compensation, with the betatron phase advance adjustment, and with the global second order chromaticity correction.

used: $2.0 \times 10^{11}, 2.5 \times 10^{11}$, and $3.0 \times 10^{11}$. The proton bunch intensity $1.5 \times 10^{11}$ is not used here because its beam loss is too small to be detected precisely even with the above hollow Gaussian distribution tracking method.

For each proton bunch intensity, we compare the relative proton beam losses in $2 \times 10^{6}$ turns without beam-beam compensation, with half beam-beam compensation, with the optimized betatron phase advances $(9 \pi, 11 \pi)$ between IP8 and the e-lens, and with the global second order chromaticity correction. Figures 17-19 show the results for the three proton bunch intensities. The vertical axis is the relative proton bunch intensity. Table II lists the calculated proton beam loss rates. For all three bunch intensities, full head-on beam-beam compensation gives a much bigger beam loss than the other beam-beam condition and therefore its beam loss is not plotted.

In Figs. 17-19, comparing the green curves with half head-on beam-beam compensation with the red curves without beam-beam compensation, half head-on beambeam compensation gives less proton particle loss in $2.0 \times 10^{6}$ for the proton bunch intensities $2.5 \times 10^{11}$ and $3.0 \times 10^{11}$. However, it does not increase the proton beam lifetime for the bunch intensity $2.0 \times 10^{11}$. The result

TABLE II. Proton beam loss rates (in units of \%/hour) under different beam-beam compensation condition.

\begin{tabular}{lccc}
\hline \hline Beam-beam & \multicolumn{3}{c}{ Proton bunch intensity } \\
\hline Condition & $2.0 \times 10^{11}$ & $2.5 \times 10^{11}$ & $3.0 \times 10^{11}$ \\
No BBC & 0.06 & 1.5 & 16.0 \\
Half BBC & 0.9 & 1.0 & 10.8 \\
Half BBC, $k \pi$ & 0.3 & 0.2 & 1.5 \\
Half BBC, $k \pi, \xi^{(2)}$ corr. & 0.15 & 0.04 & 0.4 \\
\hline \hline
\end{tabular}

TABLE III. Proton beam loss rates (in units of $\% /$ hour) in the scan of head-on beam-beam compensation strength.

\begin{tabular}{lcc}
\hline \hline Compensation & \multicolumn{2}{c}{ Proton bunch intensity } \\
\hline Strength & $2.5 \times 10^{11}$ & $3.0 \times 10^{11}$ \\
0 (no BBC) & 1.5 & 16.0 \\
0.2 & 0.5 & 2.1 \\
0.4 & 0.01 & 2.1 \\
0.5 (half BBC) & 0.04 & 0.4 \\
0.6 & 0.06 & 2.3 \\
0.8 & 23.1 & 13.4 \\
1.0 (full BBC) & 3895 & 4420 \\
\hline \hline
\end{tabular}

agrees with the dynamic aperture calculation shown in Fig. 7.

On top of half head-on beam-beam compensation, we optimize the betatron phase advances between IP8 and the center of the e-lens to be $(9 \pi, 11 \pi)$. In Figs. 17-19, the blue curves show the relative beam loss with the phase advance adjustment. For all three proton bunch intensities, the $k \pi$ betatron phase advances increase the proton beam lifetime of half beam-beam compensation. With the phase advance adjustment, the beam-beam resonance driving terms from beam-beam interaction at IP8 are better compensated with the e-lens near IP10.

We continue to correct the global second order chromaticities on top of betatron phase advance adjustment. As shown with the pink curves in Figs. 17-19, for all the three proton bunch intensities, the second order chromaticity correction further improves in the proton beam lifetime of half beam-beam compensation. Second order chromaticity correction reduces the off-momentum tune shift and the off-momentum $\beta$ beat.

Table III shows the calculated proton beam loss versus the beam-beam compensation strength. In this study, to adjust the beam-beam compensation strength, we change the electron beam intensity but keep the electron beam size constant. From Table III, the optimal compensation strengths for proton bunch intensities $2.5 \times 10^{11}$ and $3.0 \times 10^{11}$ are around half compensation, which agrees with the above dynamic aperture calculation as shown in Fig. 8.

TABLE IV. Proton beam loss rates (in units of $\% /$ hour) of half head-on beam-beam compensation in the proton working point scan.

\begin{tabular}{lcc}
\hline \hline Zero amplitude & \multicolumn{2}{c}{ Proton bunch intensity } \\
\hline tunes & $2.5 \times 10^{11}$ & $3.0 \times 10^{11}$ \\
$(28.670,29.680)$ & 0.04 & 0.4 \\
$(28.673,29.683)$ & 0.02 & 1.3 \\
$(28.676,29.686)$ & 0.63 & 5.5 \\
$(28.679,29.689)$ & 3.8 & 12.9 \\
$(28.682,29.692)$ & 10.2 & 10.0 \\
\hline \hline
\end{tabular}




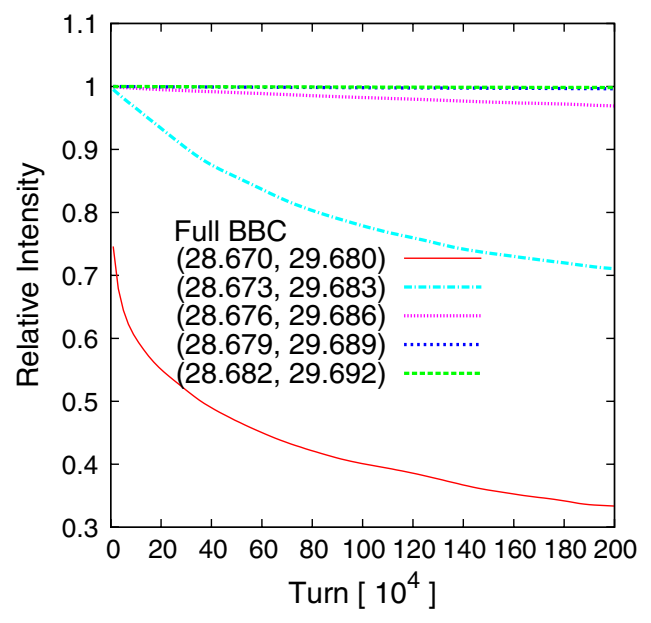

FIG. 20. Proton beam loss of full head-on beam-beam compensation in the tune scan. The bunch intensity is $2.5 \times 10^{11}$. The betatron phase adjustment and the global second order chromaticity correction are included.

Table IV shows the proton loss of half head-on beambeam compensation in the proton working point scan along the diagonal in the tune space. The betatron phase advance adjustment and second order chromaticity correction are included. In this study, the fractional vertical tune is always 0.01 higher than the fractional horizontal one. From it, for the proton bunch intensities $2.5 \times 10^{11}$ and $3.0 \times 10^{11}$, with half beam-beam compensation, a higher working point gives more proton beam loss, which also agrees with the above dynamic aperture calculation.

We also scanned the proton working point with full head-on beam-beam compensation with the proton bunch intensities $2.5 \times 10^{11}$ and $3.0 \times 10^{11}$. As an example, Fig. 20 shows the proton beam loss of full head-on beam-beam compensation with the proton bunch intensity $2.5 \times 10^{11}$. Simulation shows that full beam-beam compensation gives a smaller beam loss with a higher working point between $2 / 3$ and $7 / 10$.

For the same bunch intensity, the minimum proton beam loss rate in the tune scan with full beam-beam compensation is much higher than that with half beam-beam compensation. For example, for the proton bunch intensities $2.5 \times 10^{11}$ and $3.0 \times 10^{11}$, the minimum proton beam loss rates with full beam-beam compensation are $25.2 \%$ /hour and $60.8 \%$ /hour with the working point $(28.682,29.692)$, while the minimum proton beam loss rates with half beambeam compensation are below $1 \%$ /hour.

\section{Summary}

In this section we calculated the proton beam loss in $2 \times 10^{6}$ turns with multiparticle tracking. Simulation results show that half beam-beam compensation improves the proton beam lifetime for the bunch intensities $2.5 \times 10^{11}$ and $3.0 \times 10^{11}$. The $(9 \pi, 11 \pi)$ phase advances between IP8 and the e-lens and the global second order chromaticity correction further increase the proton beam lifetime of half beam-beam compensation. The optimum compensation strengths for the bunch intensities $2.5 \times 10^{11}$ and $3.0 \times 10^{11}$ are around half beam-beam compensation. Half beam-beam compensation is most efficient with a lower working point between $2 / 3$ and $7 / 10$. Full beam-beam compensation gives a large proton beam loss. Even with a higher working point, the proton beam loss with full beam-beam compensation is still above $25 \%$ /hour for the bunch intensities $2.5 \times 10^{11}$ and $3.0 \times 10^{11}$.

\section{SENSITIVITY STUDY}

In the following, we will study the sensitivity of head-on beam-beam compensation to the beam imperfections and beam noise [51]. We will focus on the Gaussian tail truncated electron beam, the random noise in the electron beam current, and the static and random offsets between the electron and proton beams. The baseline for this study is with the proton bunch intensity $N_{p}=2.5 \times 10^{11}$ and half beam-beam compensation. The betatron phase advance adjustment and second order chromaticity correction are included.

\section{A. Tail truncated Gaussian distribution}

In the above simulation study, we assume that the electron beam has a perfect transverse Gaussian distribution. Simulation of the electron gun for the RHIC e-lens system shows that the electron beam has a Gaussian tail cutoff at $2.8 \sigma$. This is determined by the electric field distribution on the cathode [52]. If the Gaussian tail of the electron beam is truncated at a radius $a$, the kicks on the proton are

$$
\left(\begin{array}{c}
\Delta x^{\prime} \\
\Delta y^{\prime}
\end{array}\right)_{p-e}= \begin{cases}-\frac{2 N_{e}^{*} r_{0}}{\gamma r^{2}}\left(1-e^{-r^{2} / 2 \sigma_{e}^{2}}\right)\left(\begin{array}{l}
x \\
y
\end{array}\right), & r \leqq a ; \\
-\frac{2 N_{e}^{*} r_{0}}{\gamma r^{2}}\left(1-e^{-a^{2} / 2 \sigma_{e}^{2}}\right)\left(\begin{array}{l}
x \\
y
\end{array}\right), & r>a .\end{cases}
$$

Figure 21 shows the calculated relative proton beam loss in $2 \times 10^{6}$ turns with the electron beam's Gaussian tail cutoff at $3 \sigma, 2.5 \sigma$, and $2 \sigma$. Comparing to the baseline with a prefect Gaussian distribution, the proton beam loss rate does not increase until the electron beam's Gaussian tail is cut off at $2 \sigma_{e}$. The Gaussian tail cut at $2.8 \sigma$ from the current electron gun design is acceptable.

\section{B. Noise in electron beam current}

Because of the instability of the power supplies of the electron gun, there is noise in the electron beam current. We define the relative noise ratio $\left|\Delta I / I_{0}\right|$ as the maximum amplitude of random noise in the electron current divided by the nominal current. Figure 22 shows the relative proton beam loss in $2 \times 10^{6}$ turns versus the random electron 


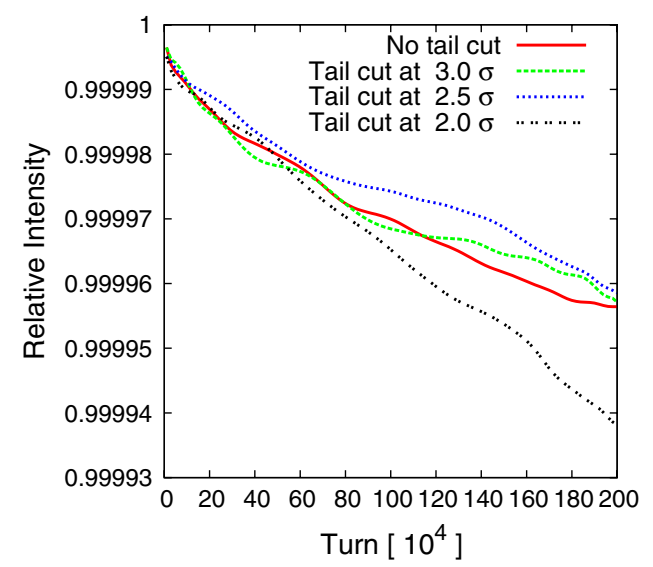

FIG. 21. Relative proton beam loss with the truncated Gaussian tail of the electron beam. Simulation of the electron gun for the RHIC e-lens system shows that the electron beam has a Gaussian tail cutoff at $2.8 \sigma$.

current noise. The proton beam loss with a random noise below $0.1 \%$ in the electron current is comparable to the baseline without current noise. $0.5 \%$ random noise in the electron beam current almost doubles the baseline's proton beam loss. In the design of the RHIC electron gun system, we require that the stability of the power supplies of the electron gun should be better than $0.1 \%$.

\section{Offsets between electron and proton beams}

Overlapping of the electron and proton beams in the e-lens plays a crucial role in the head-on beam-beam compensation. A transverse offset of electron beam with respect to the proton beam center will cause losses of beam-beam tune spread compensation and beam-beam resonance driving term cancellation. In the operation of the e-lens in RHIC, we will use beam position monitors,

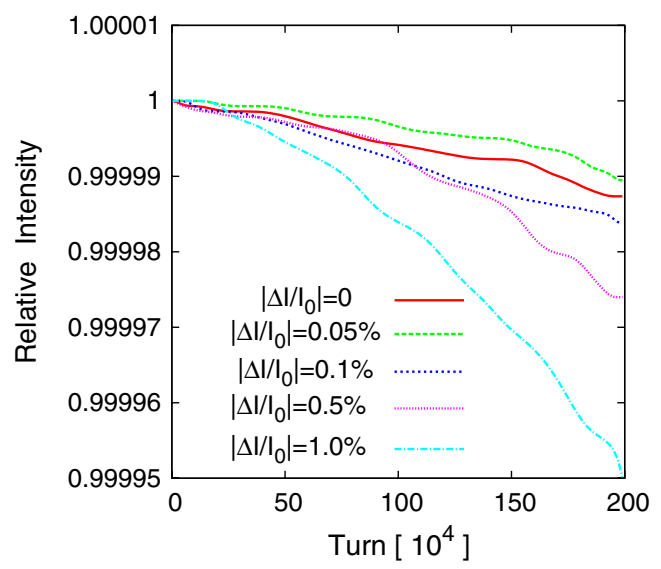

FIG. 22. Relative proton beam loss versus the random noise in the electron beam current. The relative noise ratio $\left|\Delta I / I_{0}\right|$ is defined as the maximum amplitude of random noise in the electron current divided by the nominal current.

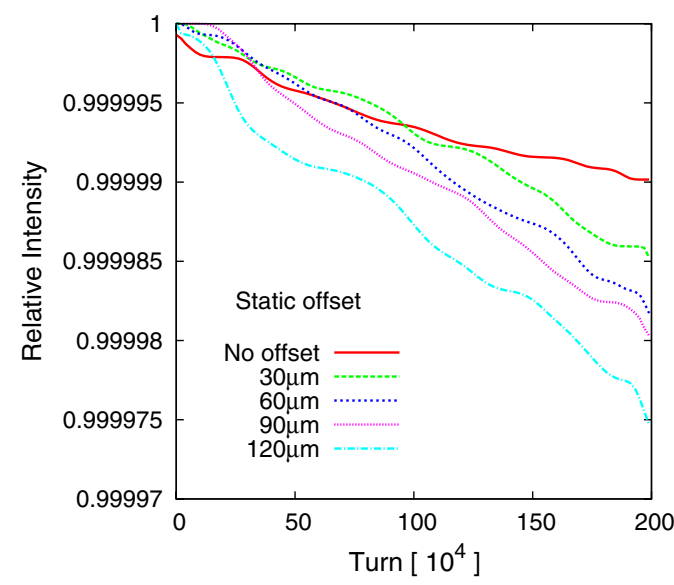

FIG. 23. Relative proton beam loss with static transverse offset between the e-lens and the proton beam in the e-lens.

together with an electron halo monitor [53] to overlap the electron and proton beams in the e-lens.

Figure 23 shows the calculated relative proton beam losses with static offsets between the electron and proton beams. In this study, the electron beam is offset transversely from the proton beam center by $30,60,90$, and $120 \mu \mathrm{m}$. From Fig. 23, when the electron beam is offset by $90 \mu \mathrm{m}$ which is about one-third of a rms beam size in the e-lens, the proton loss is doubled compared to that without offset. In the RHIC e-lens design, we set the tolerance of the static offset error to $30 \mu \mathrm{m}$ which is a tenth of a rms beam size in the e-lens.

Figure 24 shows the calculated proton beam loss with random electron beam offset. We vary the amplitude of the random offset between the electron and proton beams from 3 to $15 \mu \mathrm{m}$ with a step size of $3 \mu \mathrm{m}$. The random offset is mainly determined by the stability of the power supply of the bending magnets which bend the electron beam into the e-lenses. In the current design, we set the

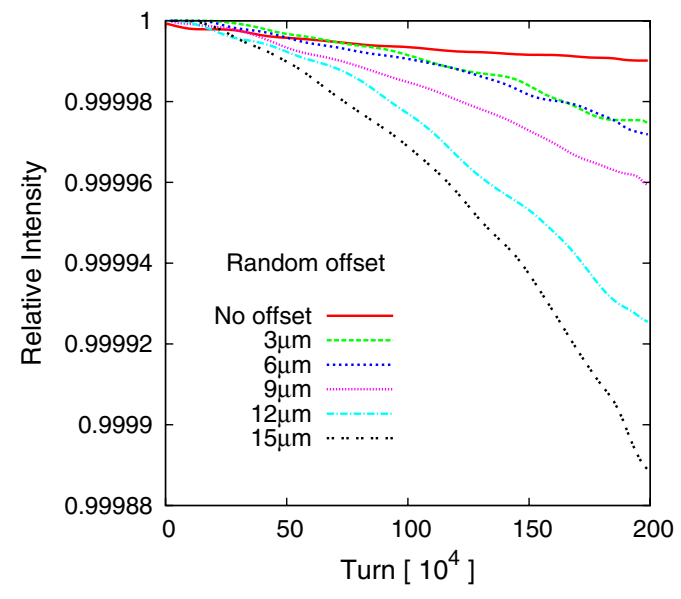

FIG. 24. Relative proton beam loss with random transverse offset between the e-lens and the proton beam in the e-lens. 
tolerance of the random offset to be $9 \mu \mathrm{m}$, which requires the stability of the bending magnet's power supply better than $0.01 \%$.

\section{Summary}

In this section, we studied the sensitivity of head-on beam-beam compensation to the electron beam's Gaussian tail cutoff, the random electron current noise, and the static and random offsets between the electron and proton beams. Based on the proton beam loss calculation in $2 \times 10^{6}$ turns, the Gaussian tail cutoff at $2.8 \sigma$ from the current electron gun design is acceptable. The random noise in the electron current should be better than $0.1 \%$. We set the tolerances of the static and random offsets between the electron and proton beams 30 and $9 \mu \mathrm{m}$, respectively.

\section{CONCLUSION}

To further increase the luminosity in RHIC polarized proton operation, it is planned to increase the proton bunch intensity from currently $1.5 \times 10^{11}$ up to $3.0 \times 10^{11}$. To hold the large beam-beam tune spread in the current tune space between $2 / 3$ and $7 / 10$ and to compensate the large beam-beam resonance driving terms, head-on beambeam compensation with e-lenses is implemented in RHIC.

With a 6D weak-strong beam-beam interaction model, we investigated the effects of head-on beam-beam compensation with electron lenses on the proton beam dynamics in RHIC polarized proton operation. Frequency map analysis, dynamic aperture, and proton beam loss rate calculations are used.

The frequency map analysis shows that there is not enough tune space between $2 / 3$ and $7 / 10$ to hold the large beam-beam tune spread when the proton bunch intensity is higher than $2.0 \times 10^{11}$. Head-on beam-beam compensation with electron lenses is effective to reduce the beambeam tune spread.

Both dynamic aperture and proton beam loss rate calculations show that half head-on beam-beam compensation increases the proton particle's dynamic aperture and beam lifetime when the proton bunch intensity is above $2.0 \times 10^{11}$. The $(9 \pi, 11 \pi)$ betatron phase advances between IP8 and the center of e-lens and the global second order chromaticity correction further improve the proton dynamic aperture and beam lifetime. Full head-on beambeam compensation reduces the proton particle's dynamic aperture and increases the proton beam loss rate. The optimum compensation for the bunch intensities $2.5 \times 10^{11}$ and $3.0 \times 10^{11}$ is around half beam-beam compensation with a slightly larger electron beam size than the proton beam size. The tune scan shows that half beambeam compensation is most effective at a lower working point between $2 / 3$ and $7 / 10$.
We also calculated the proton beam loss with various beam errors and noises. The electron beam's Gaussian tail cutoff at $2.8 \sigma$ from the current electron gun design is acceptable. The random noise in the electron current should be better than $0.1 \%$. The tolerances of the static and random offsets between the electron and proton beams are 30 and $9 \mu \mathrm{m}$, respectively.

\section{ACKNOWLEDGMENTS}

We would like to thank Y. Alexahin, M. Blaskiewicz, A. Kabel, H.-J. Kim, C. Montag, T. Roser, T. Satogata, F. Schmidt, T. Sen, V. Shiltsev, S. Tepikian, P. Thieberger, A. Valishev, S. White, and many others for stimulating discussions. This work was jointly supported by the U.S. DOE under Contract No. DE-AC02-98CH10886 and the U.S. LHC Accelerator Research Program (LARP).

[1] W. Fischer, 2005 RHIC Accelerator Physics Experiments (APEX) Workshop, 2005, Upton, NY (unpublished).

[2] Y. Luo, 2006 RHIC APEX Workshop, 2006, Upton, NY (unpublished).

[3] M. Bai et al., Phys. Rev. Lett. 96, 174801 (2006).

[4] A. Zelenski, Rev. Sci. Instrum. 81, $02 B 308$ (2010).

[5] V. Shiltsev, in The 2005 RHIC APEX Workshop, 2005, Upton, NY (unpublished).

[6] Y. Luo and W. Fischer, BNL C-AD AP Note No. 286, 2007 (unpublished).

[7] Y. Luo, W. Fischer, and N. Abreu, BNL C-AD AP Note No. 310, 2008 (unpublished).

[8] E. Tsyganov, R. Meinke, W. Nexsen, and A. Zinchenko, SSCL Report No. 519, 1993.

[9] E. Tsyganov, A. Taratin, and A. Zinchenko, Report No. JINR-E9-96-4, 1996.

[10] V. Shiltsev, V. Danilov, D. Finley, and A. Sery, Phys. Rev. ST Accel. Beams 2, 071001 (1999).

[11] V. Shiltsev, Y. Alexahin, K. Bishofberger, V. Kamerdzhiev, G. Kuznetsov, and X.-L. Zhang, Phys. Rev. Lett. 99, 244801 (2007).

[12] V. Shiltsev et al., Phys. Rev. ST Accel. Beams 11, 103501 (2008).

[13] X. Zhang, K. Bishofberger, V. Kamerdzhiev, V. Lebedev, V. Shiltsev, R. Thurman-Keup, and A. Tollestrup, Phys. Rev. ST Accel. Beams 11, 051002 (2008).

[14] W. Fischer, Y. Luo, and A. Pikin, BNL C-AD AP Note No. 359, 2009 (unpublished).

[15] E. Keil, CERN Accelerator School, Rhodes, Greece, Report No. CERN 95-06, p. 539.

[16] L. R. Evans and J. Gareyte, CERN Yellow Report No. 87-03, 1987.

[17] A. Chao, Lecture Notes on Topics in Accelerator Physics, 2001 (unpublished).

[18] Y. Papaphilippou and F. Zimmermann, Phys. Rev. ST Accel. Beams 5, 074001 (2002).

[19] T. Sen, B. Erdelyi, M. Xiao, and V. Boocha, Phys. Rev. ST Accel. Beams 7, 041001 (2004). 
[20] V. Ziemann, Nucl. Instrum. Methods Phys. Res., Sect. A 556, 45 (2006).

[21] B. V. Chirikov, Phys. Rep. 52, 263 (1979).

[22] W. Fischer, Y. Luo, and C. Montag, in Proceedings of the IPAC'10 Conference, Kyoto, Japan (ICR, Kyoto, 2010).

[23] Y. Luo et al., in Proceedings of the 23rd Particle Accelerator Conference, Vancouver, Canada, 2009 (IEEE, Piscataway, NJ, 2009).

[24] H. Kim, T. Sen, N. Abreu, and W. Fischer, Phys. Rev. ST Accel. Beams 12, 031001 (2009).

[25] R. Calaga, W. Fischer, G. Robert-Demolaize, and N. Milas, Phys. Rev. ST Accel. Beams 14, 091001 (2011).

[26] Y. Luo, S. Tepikian, W. Fischer, G. Robert-Demolaize, and D. Trbojevic, BNL Report No. C-A/AP/348, 2009 (unpublished).

[27] W. Fischer et al., in Proceedings of the CARE-HHH Workshop 2008-Scenarios for the LHC Upgrade and FAIR, Chavannes-de-Bogis, Switzerland (unpublished).

[28] C. Montag, in Proceedings of the 2011 Particle Accelerator Conference, NY, USA (IEEE, New York, 2011).

[29] Y. Luo, in Proceedings of the IPAC'10 Conference, Kyoto, Japan (Ref. [22]).

[30] R. D. Ruth, IEEE Trans. Nucl. Sci. 30, 2669 (1983).

[31] K. Hirata, H. Moshammer, and F. Ruggiero, Part. Accel. 40, 205 (1993).

[32] L. H. A. Leunissen, F. Schmidt, and G. Ripken, Phys. Rev. ST Accel. Beams 3, 124002 (2000).

[33] M. Bassetti and G. A. Erskine, Report No. CERN-ISRTH/80-06

[34] J. Laskar, Physica D (Amsterdam) 67, 257 (1993).

[35] J. Laskar and D. Robin, Part. Accel. 54, 183 (1996).

[36] Y. Papaphilippou, in Proceedings of the 18th Particle Accelerator Conference, New York, 1999 (IEEE, New York, 1999).

[37] Y. Papaphilippou and F. Zimmermann, Phys. Rev. ST Accel. Beams 2, 104001 (1999).
[38] A. Bazzani, R. Bartolini, M. Giovannozzi, W. Scandale, and E. Todesco, Part. Accel. 52, 147 (1996).

[39] J. Laskar, in Proceedings of the 20th Particle Accelerator Conference, Portland, OR, 2003 (IEEE, New York, 2003).

[40] X. Gu, Y. Luo, and W. Fischer, BNL C-AD AP Note Report No. 400, 2010 (unpublished).

[41] A. Chao and M. Tigner, Handbook of Accelerator Physics and Engineering (World Scientific, Singapore, 1998).

[42] M. Giovannozzi, W. Scandale, and E. Todesco, Part. Accel. 56, 195 (1997).

[43] GNU Scientific Library, http://www.gnu.org/software/gs1/.

[44] Y. Luo, N. Abreu, W. Fischer, and G. Robert-Demolaize, in Proceedings of the 11th European Particle Accelerator Conference, Genoa, 2008 (EPS-AG, Genoa, Italy, 2008).

[45] Y. Luo, N. Abreu, W. Fischer, R. de Maria, G. RobertDemolaize, and E. McIntosh, in Proceedings of the 23rd Particle Accelerator Conference, Vancouver, Canada, 2009 (Ref. [23]).

[46] H. J. Kim and T. Sen, in Proceedings of the IPAC'10 Conference, Kyoto, Japan (Ref. [22]).

[47] A. Valishev, in Proceedings of the IPAC'10 Conference, Kyoto, Japan (Ref. [22]).

[48] Y. Luo and W. Fischer, in Proceedings of the 46th ICFA Advanced Beam Dynamics Workshop on High-Intensity and High-Brightness Hadron Beams, Morschach, Switzerland.

[49] M. Minty et al., in Proceedings of the IPAC'10 Conference, Kyoto, Japan (Ref. [22]).

[50] R. Michiloff, 2010 RHIC Retreat Workshop, Bellport, NY (unpublished).

[51] Y. Luo et al., in Proceedings of the 2011 Particle Accelerator Conference, NY, USA (Ref. [28]).

[52] A. Pikin et al., in Proceedings of the 2011 Particle Accelerator Conference, NY, USA (Ref. [28]).

[53] P. Thieberger et al., in Proceedings of the 2011 Particle Accelerator Conference, NY, USA (Ref. [28]). 